TRANSACTIONS OF THE

AMERICAN MATHEMATICAL SOCIETY

Volume 357, Number 6, Pages 2133-2164

S 0002-9947(05)03830-4

Article electronically published on January 21, 2005

\title{
ANALYSIS OF A COUPLED SYSTEM OF KINETIC EQUATIONS AND CONSERVATION LAWS: RIGOROUS DERIVATION AND EXISTENCE THEORY VIA DEFECT MEASURES
}

\author{
M. TIDRIRI
}

\begin{abstract}
In this paper we introduce a coupled system of kinetic equations of B.G.K. type and then study its hydrodynamic limit. We obtain as a consequence the rigorous derivation and existence theory for a coupled system of kinetic equations and their hydrodynamic (conservation laws) limit. The latter is a particular case of the coupled system of Boltzmann and Euler equations. A fundamental element in this study is the rigorous derivation and justification of the interface conditions between the kinetic model and its hydrodynamic conservation laws limit, which is obtained using a new regularity theory introduced herein.
\end{abstract}

\section{INTRODUCTION}

In this paper we shall study the following coupled kinetic system:

$$
\begin{aligned}
& {\left[\partial_{t}+a(v) \cdot \partial_{x}\right] f_{\epsilon}(x, v, t)=\frac{1}{\epsilon_{l}}\left(\chi_{u_{\epsilon}(x, t)}(v)-f_{\epsilon}(x, v, t)\right) \text { in } \Omega_{l} \times V \times(0, T),} \\
& f_{\epsilon}(x, v, t)=f_{\epsilon 0}(x, v, t) \text { on } \Gamma_{1}^{-} \times(0, T), \\
& f_{\epsilon}(x, v, t)=g_{\epsilon}(x, v, t) \text { on } \Gamma_{i}^{-} \times(0, T), \\
& {\left[\partial_{t}+a(v) \cdot \partial_{x}\right] g_{\epsilon}(x, v, t)=\frac{1}{\epsilon}\left(\chi_{w_{\epsilon}(x, t)}(v)-g_{\epsilon}(x, v, t)\right) \text { in } \Omega_{g} \times V \times(0, T),} \\
& g_{\epsilon}(x, v, t)=g_{\epsilon 1}(x, v, t) \text { on } \Gamma_{2}^{-} \times(0, T), \\
& g_{\epsilon}(x, v, t)=f_{\epsilon}(x, v, t) \text { on } \Gamma_{i}^{+} \times(0, T), \\
& f_{\epsilon}(x, v, 0)=f_{\epsilon}^{0}(x, v) \text { in } \Omega_{l} \times V \quad g_{\epsilon}(x, v, 0)=g_{\epsilon}^{0}(x, v) \text { in } \Omega_{g} \times V
\end{aligned}
$$

Received by the editors January 17, 2003.

2000 Mathematics Subject Classification. Primary 35L65, 82B40.

The author was partially supported by the Air Force Office of Scientific Research under Grant F49620-99-1-0197. 
and study its relation to the coupled system

$$
\begin{aligned}
& {\left[\partial_{t}+a(v) \cdot \partial_{x}\right] f(x, v, t)=\frac{1}{\epsilon_{l}}\left(\chi_{u(x, t)}(v)-f(x, v, t)\right) \text { in } \Omega_{l} \times V \times(0, T),} \\
& f(x, v, t)=f_{0}(x, v, t) \text { on } \Gamma_{1}^{-} \times(0, T), \\
& f(x, v, t)=\chi_{w(x, t)}(v) \text { on } \Gamma_{i}^{-} \times(0, T), \\
& \partial_{t} w+\partial_{x_{i}}\left[A_{i}(w)\right]=0 \text { in } \Omega_{g} \times(0, T), \\
& \text { boundary conditions for } w \text { on } \Gamma_{2} \times(0, T) \text { and } \Gamma_{i} \times(0, T),
\end{aligned}
$$

$$
f(x, v, 0)=f^{0}(x, v) \text { in } \Omega_{l} \times V \quad w(x, 0)=w^{0}(x) \text { in } \Omega_{g} .
$$

Here, $\Omega_{l}=(0, l) \times \mathbb{R}^{d-1}, \Omega_{g}=(l, 1) \times \mathbb{R}^{d-1}$ with $0<l<1$, and $\Omega=(0,1) \times \mathbb{R}^{d-1}$ are resp. the kinetic, hydrodynamic, and physical domain and $d$ is the dimension of the spatial domain. The boundaries are defined as follows:

$$
\begin{aligned}
\Gamma_{1} & =\partial \Omega_{l} \cap \partial \Omega, \quad \Gamma_{2}=\partial \Omega_{g} \cap \partial \Omega, \quad \Gamma_{i}=\partial \Omega_{l} \cap \partial \Omega_{g} \\
\Gamma_{1}^{-} & =\left\{(x, v) \in\{0\} \times \mathbb{R}^{d-1} \times V: a(v) \cdot n_{l}(x)<0\right\} \\
\Gamma_{i}^{-} & =\left\{(x, v) \in\{l\} \times \mathbb{R}^{d-1} \times V: a(v) \cdot n_{l}(x)<0\right\} \\
\Gamma_{2}^{-} & =\left\{(x, v) \in\{1\} \times \mathbb{R}^{d-1} \times V: a(v) \cdot n_{g}(x)<0\right\} \\
\Gamma_{0 l} & =\left\{(x, v) \in \partial \Omega_{l} \times V: a(v) \cdot n_{l}(x)=0\right\} \\
\Gamma_{0 g} & =\left\{(x, v) \in \partial \Omega_{g} \times V: a(v) \cdot n_{g}(x)=0\right\}
\end{aligned}
$$

where $n_{l}$ resp. $n_{g}$ denotes the exterior unit normal vector to $\Omega_{l}$ resp. $\Omega_{g}$. In the sequel we will denote by $n$ any exterior unit normal vector; it will be clear from the context which normal we mean. We shall assume that $\Gamma_{0 l}$ has measure 0 in $\partial \Omega_{l} \times V$ for the measure $d \gamma d v$ and that $\Gamma_{0 g}$ has measure 0 in $\partial \Omega_{g} \times V$ for the measure $d \gamma d v$.

The boundary conditions in (12) for the conservation laws are prescribed on a part of $\Gamma_{i}$ resp. $\Gamma_{2}$. These boundary conditions together with those in (10) will be made precise in Definition 3.1. The set $V=\mathbb{R}$ is the velocity domain and $T$ is a positive constant.

The function $f$ describes the microscopic density of particles at $(x, t)$ with velocity $v$ in the kinetic domain. The function $w$ describes the local density of particles at $(x, t)$ in the hydrodynamic domain.

The functions $f_{\epsilon}$ and $f$ resp. $g_{\epsilon}$ describe the microscopic density of particles at $(x, t)$ with velocity $v$ in the kinetic resp. hydrodynamic domain. The physical parameter $\epsilon_{l}$ resp. $\epsilon>0$ is the microscopic scale in the kinetic resp. hydrodynamic domain. The functions $f_{\epsilon}^{0}$ and $g_{\epsilon}^{0}$ are the initial data while $f_{\epsilon 0}, f_{0}$, and $g_{\epsilon 1}$ are the boundary data. The boundary conditions in Eqs. (3) and (6) describe the transmission conditions between the two kinetic equations. The local densities of particles $u_{\epsilon}, u$, and $w_{\epsilon}$ at $(x, t)$ are related to the microscopic densities $f_{\epsilon}, f$, and $g_{\epsilon}$ by $u_{\epsilon}(x, t)=\int_{V} f_{\epsilon}(x, v, t) d v, u(x, t)=\int_{V} f(x, v, t) d v$, and $w_{\epsilon}(x, t)=\int_{V} g_{\epsilon}(x, v, t) d v$. The boundary conditions in (12) also involve $w_{1}$ which is a given boundary data; see Definition 3.1 below. The boundary conditions in Eqs. (10) and (12) describe the transmission conditions between the kinetic and the hydrodynamic domain. Let $A=\left(A_{i}\right)_{1 \leq i \leq d}$ with components related to $a_{i}(\cdot)$ by $a_{i}(\cdot)=A_{i}^{\prime}(\cdot), i=1, \cdots, d$. We 
assume that the functions $a_{i}(\cdot) \in C^{2, \alpha}$ for some $\alpha>0$. The collisions in the kinetic equations are given by the nonlinear kernel in the right-hand side of Eqs. (10), (4), and (8) in which $\chi_{u}(v)$ is the signature of $u$ defined by

$$
\chi_{u}(v)= \begin{cases}1 & \text { if } 0<v \leq u \\ -1 & \text { if } u \leq v<0 \\ 0 & \text { otherwise }\end{cases}
$$

This problem is a particular case of the more general coupled systems consisting of Boltzmann equations and their hydrodynamic limits introduced and studied in [21, 22, 23] (see also the references therein). These problems are introduced in order to solve the problem of intermediate regimes in gas dynamics. Their practical importance in real life applications has been successfully proven in 21 . 2223 (see also the references therein). A complete mathematical theory of these methodologies in the case of a Carleman simplified Boltzmann model has been developed by the author in 23. Here we shall study a nonlinear Boltzmann model which is a bit more general. The study of the hydrodynamic limit of the model in (4) in full space was done by Perthame and Tadmor [16, Perthame [15], and the author [26, 27]. They proved that this model converges as the microscopic scale goes to 0 to a conservation law of the form in (11). In the case of bounded domain such study is performed by the author in [26, 27]. Further studies using this model and closely related models can be found in the references in [15].

Here we study this nonlinear model in the context of domains with boundaries and coupling of Boltzmann equations and their hydrodynamic limits. More precisely we shall study the hydrodynamic limit of the kinetic system (11)-(17) as the microscopic scale $\epsilon \rightarrow 0$ in $\Omega_{g}$. As a consequence we shall rigorously derive and establish the existence theory of the system (8)-(13). Systems (1)-(7) have been introduced by the author in [25], where he established the hydrodynamic limit analysis of this kinetic system and rigorously derived system (8)-(13). In [25], the author made the additional assumption that the initial data are in $B V_{l o c}$. Here we shall remove such an assumption, however, we shall make a nondegeneracy assumption on the flux.

A fundamental element in this study is the rigorous derivation of the interface conditions between the kinetic model and hydrodynamic conservation laws limit. This is obtained using a new regularity theory developed here. Besides its intrinsic merit, we expect that the study developed here of the interfacial kinetichydrodynamic problem may provide methods and ideas that might help solve other problems involving interfaces between physical phenomena of a different scale and nature.

In the next section we shall study the kinetic system and give various information about its solution. These results have been proved in 25]. We shall also give some results about generalized Green and Gauss-Green formulas for bounded Radon measures and about the weak traces for a particular class of functions. In Section 3 , we shall introduce the kinetic formulation of the coupled system (8)-(13) and prove its equivalence to system (8)-(13) in the sense of Definitions 3.1 and 3.2. In the fourth section we prove that the solution of the kinetic system converges to a solution of the system given by the kinetic formulation. Finally, in the last section we prove that the system (8)-(13) has an entropic solution in the sense of Definition 3.1 . 


\section{Coupled System of Kinetic EQuations}

In this section we shall present some basic results about the kinetic system (11)(7). These results and their proofs were obtained in [25]. However, for the sake of completeness and independent reading we will give below the proofs of such results. We begin with stating a result about the well-posedness of the kinetic system (1)-(7). We then establish various properties of the solution, including $L^{\infty}$, $L^{1}$, and $B V_{l o c}$ uniform estimates. These estimates will be used for the study of the hydrodynamic limit of system (1)-(7) as $\epsilon \rightarrow 0$ in $\Omega_{g}$. We shall also use the following notations:

$$
\begin{aligned}
\Omega_{0} & =\left\{(x, v, t) \in \Omega_{l} \times V \times(0, T): x_{1}-a_{1}(v) t<0\right\}, \\
\Omega_{a} & =\left\{(x, v, t) \in \Omega_{l} \times V \times(0, T): x_{1}-a_{1}(v) t>l\right\}, \\
\Omega_{0 a} & =\left\{(x, v, t) \in \Omega_{l} \times V \times(0, T): 0<x_{1}-a_{1}(v) t<l\right\}, \\
\Omega_{1} & =\left\{(x, v, t) \in \Omega_{g} \times V \times(0, T): x_{1}-a_{1}(v) t>1\right\}, \\
\Omega_{a 1} & =\left\{(x, v, t) \in \Omega_{g} \times V \times(0, T): l<x_{1}-a_{1}(v) t<1\right\}, \\
\Omega_{a a} & =\left\{(x, v, t) \in \Omega_{g} \times V \times(0, T): x_{1}-a_{1}(v) t<l\right\},
\end{aligned}
$$

where $x=\left(x_{1}, x_{\star}\right)$.

2.1. Existence theory. We have the following existence and uniqueness result.

Theorem 2.1. Assume that

$$
\begin{array}{cl}
f_{\epsilon}^{0} \in L^{1}\left(\Omega_{l} \times V\right), & g_{\epsilon}^{0} \in L^{1}\left(\Omega_{g} \times V\right), \\
a(v) \cdot n_{l} f_{\epsilon 0} \in L^{1}\left(\Gamma_{1}^{-} \times(0, T)\right), & a(v) \cdot n_{g} g_{\epsilon 1} \in L^{1}\left(\Gamma_{2}^{-} \times(0, T)\right) .
\end{array}
$$

Then

(i) The coupled system (1)-(7) has a unique solution $\left(f_{\epsilon}, g_{\epsilon}\right)$ in $L^{\infty}\left([0, T] ; L^{1}\left(\Omega_{l} \times V\right)\right) \times L^{\infty}\left([0, T] ; L^{1}\left(\Omega_{g} \times V\right)\right)$. Moreover, $\left(f_{\epsilon}, g_{\epsilon}\right)$ satisfies the integral representation

$$
\begin{array}{ll}
\text { In } \Omega_{0} \quad & f_{\epsilon}(x, v, t)=\exp \left(-x_{1} / \epsilon_{l} a_{1}(v)\right) f_{\epsilon}\left(\left(0, x_{\star}-\frac{x_{1}}{a_{1}(v)} a_{\star}(v)\right), v, t-x_{1} / a_{1}(v)\right) \\
& +\frac{1}{\epsilon_{l}} \int_{t-x_{1} / a_{1}(v)}^{t} e^{(s-t) / \epsilon_{l}} \chi_{u_{\epsilon}(x(s), s)}(v) d s . \\
& f_{\epsilon}(x, v, t)=g_{\epsilon}\left(\left(l, x_{\star}+\frac{l-x_{1}}{a_{1}(v)} a_{\star}(v)\right), v, t-\frac{x_{1}-l}{a_{1}(v)}\right) \exp \left(-\left(x_{1}-l\right) / \epsilon_{l} a_{1}(v)\right) \\
& +\frac{1}{\epsilon_{l}} \int_{t-\frac{x_{1}-l}{a_{1}(v)}}^{t} e^{(s-t) / \epsilon_{l}} \chi_{u_{\epsilon}(x(s), s)}(v) d s . \\
\text { In } \Omega_{0 a} \quad f_{\epsilon}(x, v, t)=f_{\epsilon}^{0}(x-a(v) t, v) \exp \left(-t / \epsilon_{l}\right)+\frac{1}{\epsilon_{l}} \int_{0}^{t} e^{(s-t) / \epsilon_{l}} \chi_{u_{\epsilon}(x(s), s)}(v) d s . \\
\text { In } \Omega_{a a} \quad g_{\epsilon}(x, v, t)=f_{\epsilon}\left(\left(l, x_{\star}+\frac{l-x_{1}}{a_{1}(v)} a_{\star}(v)\right), v, t-\frac{x_{1}-l}{a_{1}(v)}\right) \exp \left(-\left(x_{1}-l\right) /\left(a_{1}(v) \epsilon\right)\right) \\
\quad+\frac{1}{\epsilon} \int_{t-\frac{x_{1}-l}{a_{1}(v)}}^{t} e^{(s-t) / \epsilon} \chi_{w_{\epsilon}(x(s), s)}(v) d s .
\end{array}
$$




$$
\begin{aligned}
\text { In } \Omega_{a 1} \quad g_{\epsilon}(x, v, t) & =g_{\epsilon}^{0}(x-a(v) t, v) \exp (-t / \epsilon)+\frac{1}{\epsilon} \int_{0}^{t} e^{(s-t) / \epsilon} \chi_{w_{\epsilon}(x(s), s)}(v) d s . \\
\text { In } \Omega_{1} \quad g_{\epsilon}(x, v, t) & =g_{\epsilon 1}\left(\left(1, x_{\star}+\frac{1-x_{1}}{a_{1}(v)} a_{\star}(v)\right), v, t-\frac{x_{1}-1}{a_{1}(v)}\right) \exp \left(\left(1-x_{1}\right) / \epsilon a_{1}(v)\right) \\
+\frac{1}{\epsilon} \int_{t-\frac{x_{1}-1}{a_{1}(v)}}^{t} & e^{(s-t) / \epsilon} \chi_{w_{\epsilon}(x(s), s)}(v) d s,
\end{aligned}
$$

where $x(s)=x+(s-t) a(v), x=\left(x_{1}, x_{\star}\right)$, and $a(v)=\left(a_{1}(v), a_{\star}(v)\right)$.

On $\Gamma_{i}^{-} \times(0, T)$, we have:

For $l<l-t a_{1}(v)<1$,

$$
\begin{aligned}
g_{\epsilon}\left(\left(l, x_{\star}\right), v, t\right)= & e^{-t / \epsilon} g_{\epsilon}^{0}\left(\left(l-t a_{1}(v), x_{\star}-t a_{\star}(v)\right), v\right) \\
& +\frac{1}{\epsilon} \int_{0}^{t} e^{(s-t) / \epsilon} \chi_{w_{\epsilon}(x(s), s)}(v) d s .
\end{aligned}
$$

For $1<l-t a_{1}(v)$,

$$
\begin{aligned}
g_{\epsilon}\left(\left(l, x_{\star}\right), v, t\right)= & e^{(1-l) /\left(\epsilon a_{1}(v)\right)} g_{\epsilon}\left(\left(1, x_{\star}+\frac{1-l}{a_{1}(v)} a_{\star}(v)\right), v, t+\frac{1-l}{a_{1}(v)}\right) \\
& +\frac{1}{\epsilon} \int_{t+\frac{1-l}{a_{1}(v)}}^{t} e^{(s-t) / \epsilon} \chi_{w_{\epsilon}(x(s), s)}(v) d s .
\end{aligned}
$$

We also have a similar formula for $f_{\epsilon}$ on $\Gamma_{i}^{+} \times(0, T)$.

(ii) Let $\left(f_{\epsilon}, g_{\epsilon}\right)$ and $\left(F_{\epsilon}, G_{\epsilon}\right)$ be two solutions of (11)-(17) with corresponding densities $u_{\epsilon}(x, t)=\int_{V} f_{\epsilon}(x, v, t) d v, w_{\epsilon}(x, t)=\int_{V} g_{\epsilon}(x, v, t) d v$ and $U_{\epsilon}(x, t)=$ $\int_{V} F_{\epsilon}(x, v, t) d v, W_{\epsilon}(x, t)=\int_{V} G_{\epsilon}(x, v, t) d v$; and let $f_{\epsilon}^{0}, g_{\epsilon}^{0}, f_{\epsilon 0}, g_{\epsilon 1}$ resp. $F_{\epsilon}^{0}, G_{\epsilon}^{0}$, $F_{\epsilon 0}, G_{\epsilon 1}$ denote the corresponding data. Then for any $0 \leq t \leq T$, we have

$$
\begin{aligned}
& \quad\left\|f_{\epsilon}-F_{\epsilon}\right\|_{L^{1}\left(\Omega_{l} \times V\right)}+\left\|g_{\epsilon}-G_{\epsilon}\right\|_{L^{1}\left(\Omega_{g} \times V\right)}+\left\|a(v) \cdot n\left(f_{\epsilon}-F_{\epsilon}\right)\right\|_{L^{1}\left(\Gamma_{1}^{+} \times(0, t)\right)} \\
& \quad+\left\|a(v) \cdot n\left(g_{\epsilon}-G_{\epsilon}\right)\right\|_{L^{1}\left(\Gamma_{2}^{+} \times(0, t)\right)} \\
& \leq\left\|f_{\epsilon}^{0}-F_{\epsilon}^{0}\right\|_{L^{1}\left(\Omega_{l} \times V\right)}+\left\|g_{\epsilon}^{0}-G_{\epsilon}^{0}\right\|_{L^{1}\left(\Omega_{g} \times V\right)}+\left\|a(v) \cdot n\left(f_{\epsilon 0}-F_{\epsilon 0}\right)\right\|_{L^{1}\left(\Gamma_{1}^{-} \times(0, t)\right)} \\
& \quad+\left\|a(v) \cdot n\left(g_{\epsilon 1}-G_{\epsilon 1}\right)\right\|_{L^{1}\left(\Gamma_{2}^{-} \times(0, t)\right)} .
\end{aligned}
$$

Proof of Theorem 2.1. We begin by proving (ii), which states a result about the uniqueness and the continuous dependence of the solution on the data. The idea of the proof is to use a combination of the author's method [20, 21] and ideas from 10.

The function $F_{\epsilon}$ satisfies an equation similar to Eq. (1). Subtracting this equation from Eq. (1), and multiplying the resulting equation by

$$
\varphi_{1}=\operatorname{sgn}^{\mu}\left(f_{\epsilon}-F_{\epsilon}\right) \psi_{1}(x, t)
$$

with $x \operatorname{sgn}^{\mu}(x) \geq 0, x \in \mathbb{R}$, where $\psi_{1}$ is a nonnegative test function and $\operatorname{sgn}^{\mu}$ is a regularization of sign function, and integrating by parts, and letting $\mu \rightarrow 0$, we 
obtain

$$
\begin{aligned}
& \int_{\Omega_{l} \times V}\left(\left|f_{\epsilon}-F_{\epsilon}\right| \psi_{1}\right)(\cdot, \cdot, t)+\int_{\Gamma_{i}^{-} \times(0, t)} a(v) \cdot n\left|f_{\epsilon}-F_{\epsilon}\right| \psi_{1} \\
& +\int_{\Gamma_{i}^{+} \times(0, t)} a(v) \cdot n\left|f_{\epsilon}-F_{\epsilon}\right| \psi_{1}+\int_{\Gamma_{1}^{+} \times(0, t)} a(v) \cdot n\left|f_{\epsilon}-F_{\epsilon}\right| \psi_{1} \\
& +\frac{1}{\epsilon_{l}} \int_{\Omega_{l} \times V \times(0, t)}\left|f_{\epsilon}-F_{\epsilon}\right| \psi_{1} \\
& =\frac{1}{\epsilon_{l}} \int_{\Omega_{l} \times V \times(0, t)}\left(\chi_{u_{\epsilon}}-\chi_{U_{\epsilon}}\right) \operatorname{sgn}\left(f_{\epsilon}-F_{\epsilon}\right) \psi_{1}+\int_{\Omega_{l} \times V}\left(\left|f_{\epsilon}-F_{\epsilon}\right| \psi_{1}\right)(\cdot, \cdot, 0)
\end{aligned}
$$

$$
-\int_{\Gamma_{1}^{-} \times(0, t)} a(v) \cdot n\left|f_{\epsilon 0}-F_{\epsilon 0}\right| \psi_{1}+\int_{\Omega_{l} \times V \times(0, t)}\left(\partial_{t}+a(v) \cdot \partial_{x}\right)\left(\psi_{1}\right)\left|f_{\epsilon}-F_{\epsilon}\right| .
$$

Using the properties of $\chi$, this yields

$$
\begin{aligned}
& \int_{\Omega_{l} \times V}\left(\left|f_{\epsilon}-F_{\epsilon}\right| \psi_{1}\right)(\cdot, \cdot, t)+\int_{\Gamma_{i}^{-} \times(0, t)} a(v) \cdot n\left|f_{\epsilon}-F_{\epsilon}\right| \psi_{1} \\
& +\int_{\Gamma_{i}^{+} \times(0, t)} a(v) \cdot n\left|f_{\epsilon}-F_{\epsilon}\right| \psi_{1}+\int_{\Gamma_{1}^{+} \times(0, t)} a(v) \cdot n\left|f_{\epsilon}-F_{\epsilon}\right| \psi_{1} \\
\leq & \int_{\Omega_{l} \times V}\left(\left|f_{\epsilon}-F_{\epsilon}\right| \psi_{1}\right)(\cdot, \cdot, 0)-\int_{\Gamma_{1}^{-} \times(0, t)} a(v) \cdot n\left|f_{\epsilon 0}-F_{\epsilon 0}\right| \psi_{1} \\
& +\int_{\Omega_{l} \times V \times(0, t)}\left(\partial_{t}+a(v) \cdot \partial_{x}\right)\left(\psi_{1}\right)\left|f_{\epsilon}-F_{\epsilon}\right| .
\end{aligned}
$$

Similarly, multiplying Eq. (4) by $\varphi_{2}=\operatorname{sgn}^{\mu}\left(g_{\epsilon}-G_{\epsilon}\right) \psi_{2}(x, t)$ with $\psi_{2}$ a nonnegative test function in $C^{1}\left(\bar{\Omega}_{g} \times[0, T]\right)$, and proceeding as above, we obtain an inequation for $g_{\epsilon}-G_{\epsilon}$ similar to Eq. (17). Adding this inequation to Eq. (17), taking $\psi_{1}$ and $\psi_{2}$ such that $\psi_{1}=\psi_{2}$ on $\Gamma_{i} \times(0, T)$, and using the transmission conditions, we obtain

$$
\begin{aligned}
& \int_{\Omega_{l} \times V}\left(\left|f_{\epsilon}-F_{\epsilon}\right| \psi_{1}\right)(\cdot, \cdot, t)+\int_{\Omega_{g} \times V}\left(\left|g_{\epsilon}-G_{\epsilon}\right| \psi_{2}\right)(\cdot, \cdot, t) \\
& +\int_{\Gamma_{1}^{+} \times(0, t)} a(v) \cdot n\left|f_{\epsilon}-F_{\epsilon}\right| \psi_{1}+\int_{\Gamma_{2}^{+} \times(0, t)} a(v) \cdot n\left|g_{\epsilon}-G_{\epsilon}\right| \psi_{2} \\
\leq & \int_{\Omega_{l} \times V}\left(\left|f_{\epsilon}-F_{\epsilon}\right| \psi_{1}\right)(\cdot, \cdot, 0)+\int_{\Omega_{g} \times V}\left(\left|g_{\epsilon}-G_{\epsilon}\right| \psi_{2}\right)(\cdot, \cdot, 0) \\
& -\int_{\Gamma_{1}^{-} \times(0, t)} a(v) \cdot n\left|f_{\epsilon 0}-F_{\epsilon 0}\right| \psi_{1}+\int_{\Omega_{l} \times V \times(0, t)}\left(\partial_{t}+a(v) \cdot \partial_{x}\right)\left(\psi_{1}\right)\left|f_{\epsilon}-F_{\epsilon}\right| \\
& -\int_{\Gamma_{2}^{-} \times(0, t)} a(v) \cdot n\left|g_{\epsilon 1}-G_{\epsilon 1}\right| \psi_{2}+\int_{\Omega_{g} \times V \times(0, t)}\left(\partial_{t}+a(v) \cdot \partial_{x}\right)\left(\psi_{2}\right)\left|g_{\epsilon}-G_{\epsilon}\right| .
\end{aligned}
$$

Now taking $\psi_{1}(t)=\psi_{2}(t)=1$ yields the estimate (15). 
To prove the existence of a solution to the kinetic system, we introduce the following iterations:

$$
\begin{aligned}
& {\left[\partial_{t}+a(v) \cdot \partial_{x}\right] f_{\epsilon}^{n+1}(x, v, t)=\frac{1}{\epsilon_{l}}\left(\chi_{u_{\epsilon}^{n}(x, t)}(v)-f_{\epsilon}^{n+1}(x, v, t)\right) \text { in } \Omega_{l} \times V \times(0, T),} \\
& f_{\epsilon}^{n+1}(x, v, t)=f_{\epsilon 0}(x, v, t) \text { on } \Gamma_{1}^{-} \times(0, T), \\
& f_{\epsilon}^{n+1}(x, v, t)=g_{\epsilon}^{n+1}(x, v, t) \text { on } \Gamma_{i}^{-} \times(0, T), \\
& {\left[\partial_{t}+a(v) \cdot \partial_{x}\right] g_{\epsilon}^{n+1}(x, v, t)=\frac{1}{\epsilon}\left(\chi_{w_{\epsilon}^{n+1}(x, t)}(v)-g_{\epsilon}^{n+1}(x, v, t)\right) \text { in } \Omega_{g} \times V \times(0, T),} \\
& g_{\epsilon}^{n+1}(x, v, t)=g_{\epsilon 0}(x, v, t) \text { on } \Gamma_{2}^{-} \times(0, T), \\
& g_{\epsilon}^{n+1}(x, v, t)=f_{\epsilon}^{n+1}(x, v, t) \text { on } \Gamma_{i}^{+} \times(0, T), \\
& f_{\epsilon}^{n+1}(x, v, 0)=f_{\epsilon}^{0}(x, v) \quad g_{\epsilon}^{n+1}(x, v, 0)=g_{\epsilon}^{0}(x, v) .
\end{aligned}
$$

Using (16) in the present context with $f_{\epsilon}=f_{\epsilon}^{n+1}$ and $F_{\epsilon}=f_{\epsilon}^{m+1}$, and the properties of $\chi$, we obtain

$$
\begin{aligned}
& \int_{\Omega_{l} \times V}\left(\left|f_{\epsilon}-F_{\epsilon}\right| \psi_{1}\right)(\cdot, \cdot, t)+\int_{\Gamma_{i}^{-} \times(0, t)} a(v) \cdot n\left|f_{\epsilon}-F_{\epsilon}\right| \psi_{1} \\
& +\int_{\Gamma_{i}^{+} \times(0, t)} a(v) \cdot n\left|f_{\epsilon}-F_{\epsilon}\right| \psi_{1} \\
& +\int_{\Gamma_{1}^{+} \times(0, t)} a(v) \cdot n\left|f_{\epsilon}-F_{\epsilon}\right| \psi_{1}+\frac{1}{\epsilon_{l}} \int_{\Omega_{l} \times V \times(0, t)}\left|f_{\epsilon}-F_{\epsilon}\right| \psi_{1} \\
(19) \leq & \frac{1}{\epsilon_{l}} \int_{\Omega_{l} \times V \times(0, t)}\left|f_{\epsilon}^{n}-f_{\epsilon}^{m}\right| \psi_{1}+\int_{\Omega_{l} \times V \times(0, t)}\left(\partial_{t}+a(v) \cdot \partial_{x}\right)\left(\psi_{1}\right)\left|f_{\epsilon}-F_{\epsilon}\right| .
\end{aligned}
$$

Similarly taking $g_{\epsilon}=g_{\epsilon}^{n+1}$ and $G_{\epsilon}=g_{\epsilon}^{m+1}$, and using the properties of $\chi$, we obtain

$$
\begin{aligned}
& \int_{\Omega_{g} \times V}\left(\left|g_{\epsilon}-G_{\epsilon}\right| \psi_{2}\right)(\cdot, \cdot, t)+\int_{\Gamma_{i}^{+} \times(0, t)} a(v) \cdot n_{g}\left|g_{\epsilon}-G_{\epsilon}\right| \psi_{2} \\
& +\int_{\Gamma_{i}^{-} \times(0, t)} a(v) \cdot n_{g}\left|g_{\epsilon}-G_{\epsilon}\right| \psi_{2}+\int_{\Gamma_{2}^{+} \times(0, t)} a(v) \cdot n_{g}\left|g_{\epsilon}-G_{\epsilon}\right| \psi_{2} \\
\leq & \int_{\Omega_{g} \times V \times(0, t)}\left(\partial_{t}+a(v) \cdot \partial_{x}\right)\left(\psi_{2}\right)\left|g_{\epsilon}-G_{\epsilon}\right| .
\end{aligned}
$$

Taking $\psi_{1}=\psi_{2}=e^{-\frac{\alpha}{\epsilon_{l}} s}, 0 \leq s \leq t$, with $\alpha$ a positive constant, adding the equations in (19) and (20), and using the transmission conditions and the properties of $\chi$, we 
obtain

$$
\begin{aligned}
& \int_{\Omega_{l} \times V}\left(\left|f_{\epsilon}-F_{\epsilon}\right| \psi_{1}\right)(\cdot, \cdot, t)+\int_{\Omega_{g} \times V}\left(\left|g_{\epsilon}-G_{\epsilon}\right| \psi_{2}\right)(\cdot, \cdot, t) \\
& +\int_{\Omega_{l} \times V \times(0, t)} \frac{1+\alpha}{\epsilon_{l}} \psi_{1}\left|f_{\epsilon}-F_{\epsilon}\right| \\
& +\int_{\Gamma_{1}^{+} \times(0, t)} a(v) \cdot n\left|f_{\epsilon}-F_{\epsilon}\right| \psi_{1}+\int_{\Gamma_{2}^{+} \times(0, t)} a(v) \cdot n_{g}\left|g_{\epsilon}-G_{\epsilon}\right| \psi_{2} \\
& +\int_{\Omega_{g} \times V \times(0, t)} \frac{\alpha}{\epsilon_{l}} \psi_{2}\left|g_{\epsilon}-G_{\epsilon}\right| \leq \frac{1}{\epsilon_{l}} \int_{\Omega_{l} \times V \times(0, t)}\left|f_{\epsilon}^{n}-f_{\epsilon}^{m}\right| \psi_{1} .
\end{aligned}
$$

Hence we obtain

$$
\int_{\Omega_{l} \times V \times(0, t)} \psi_{1}\left|f_{\epsilon}^{n+1}-f_{\epsilon}^{m+1}\right| \leq \frac{1}{1+\alpha} \int_{\Omega_{l} \times V \times(0, t)}\left|f_{\epsilon}^{n}-f_{\epsilon}^{m}\right| \psi_{1} .
$$

This and a reuse of (21) proves that the iterations are contracted to a fixed point in $L^{\infty}\left((0, T) ; L^{1}\left(\Omega_{l} \times V\right)\right) \times L^{\infty}\left((0, T) ; L^{1}\left(\Omega_{g} \times V\right)\right)$, which satisfies Eqs. (1) and (4) and also the boundary and initial conditions (22)-(3) and (5)-(7) (use the arbitrariness of the functions $\psi_{1}$ and $\psi_{2}$ together with the above convergence). We also infer from the inequality (15) that the solution $\left(f_{\epsilon}, g_{\epsilon}\right)$ depends continuously on the initial and boundary data. The fact that $\left(f_{\epsilon}, g_{\epsilon}\right)$ satisfies the integral representation is a direct consequence of the characteristic method. The proof of Theorem 2.1 is now finished.

Remark 2.1. The proof of the well-posedness of the uncoupled kinetic equations relies on a fixed method based on the integral representation of the solution (see [16, 26]). Because of the transmission boundary conditions, a direct application of the integral representation fails to give contracting operators. It is possible, however, to prove the continuous dependence of the solution on the data using an alternative form of the integral representation.

2.2. Kinetic entropy. We shall prove the following entropy inequality for the solution of the kinetic system.

Theorem 2.2. The solution to the kinetic system satisfies the relations

$$
\begin{aligned}
& -\int_{\Omega_{l} \times V \times(0, T)}\left(\partial_{t}+a(v) \cdot \partial_{x}\right)\left(\psi_{1}\right)\left|f_{\epsilon}-\chi_{k}\right|+\int_{\Gamma_{i}^{-} \times(0, T)} a(v) \cdot n \psi_{1}\left|g_{\epsilon}-\chi_{k}\right| \\
& +\int_{\Gamma_{1}^{-} \times(0, T)} a(v) \cdot n \psi_{1}\left|f_{\epsilon 0}-\chi_{k}\right| \\
= & -\int_{\Gamma_{i}^{+} \times(0, T)} a(v) \cdot n \psi_{1}\left|f_{\epsilon}-\chi_{k}\right|-\int_{\Gamma_{1}^{+} \times(0, T)} a(v) \cdot n \psi_{1}\left|f_{\epsilon}-\chi_{k}\right| \\
& +\frac{1}{\epsilon_{l}} \int_{\Omega_{l} \times V \times(0, T)}\left(\chi_{u_{\epsilon}}-f_{\epsilon}\right) \psi_{1} \operatorname{sgn}\left(f_{\epsilon}-\chi_{k}\right)
\end{aligned}
$$


and

$$
\begin{aligned}
(24) & -\int_{\Omega_{g} \times V \times(0, T)}\left(\partial_{t}+a(v) \cdot \partial_{x}\right)\left(\psi_{2}\right)\left|g_{\epsilon}-\chi_{k}\right|+\int_{\Gamma_{i}^{+} \times(0, T)} a(v) \cdot n_{g} \psi_{2}\left|f_{\epsilon}-\chi_{k}\right| \\
& +\int_{\Gamma_{2}^{-} \times(0, T)} a(v) \cdot n_{g} \psi_{2}\left|g_{\epsilon 1}-\chi_{k}\right| \leq 0 \\
& \forall \psi_{1} \in C_{0}^{1}\left(\bar{\Omega}_{l} \times V \times(0, T)\right), \forall \psi_{2} \in C_{0}^{1}\left(\bar{\Omega}_{g} \times(0, T)\right), \quad \psi_{2} \geq 0, \forall k \in \mathbb{R} .
\end{aligned}
$$

Proof. Multiplying Eq. (1) by $\varphi_{1}=\operatorname{sgn}^{\mu}\left(f_{\epsilon}-\chi_{k}\right) \psi_{1}(x, t)$ and Eq. (4) by $\varphi_{2}=$ $\operatorname{sgn}^{\mu}\left(g_{\epsilon}-\chi_{k}\right) \psi_{2}(x, t)$ (see the proof of Theorem 2.1) and proceeding as in the proof of Theorem 2.1, we obtain Eq. (23) and similar equation for $g_{\epsilon}$. Now using the properties of $\chi$, we obtain Eq. (24). The theorem is now proved.

2.3. Basic estimates of the solution. We shall state and prove here some basic estimates for the solution of the kinetic system. We begin with $L^{\infty}$ estimates of $f_{\epsilon}$ and $g_{\epsilon}$.

Lemma 2.1. Assume that

$$
\begin{gathered}
\left\|f_{\epsilon 0}\right\|_{L^{\infty}\left(\Gamma_{1}^{-} \times[0, T]\right)}<C_{1},\left\|g_{\epsilon}^{0}\right\|_{L^{\infty}\left(\Omega_{g} \times V\right)}<C_{2}, \\
\left\|f_{\epsilon}^{0}\right\|_{L^{\infty}\left(\Omega_{l} \times V\right)}<C_{3},\left\|g_{\epsilon 1}\right\|_{L^{\infty}\left(\Gamma_{2}^{-} \times[0, T]\right)}<C_{4}
\end{gathered}
$$

with $C_{1}, C_{2}, C_{3}$, and $C_{4}$ independent of $\epsilon$. Then $f_{\epsilon}$ and $g_{\epsilon}$ are uniformly bounded resp. in $L^{\infty}\left(\Omega_{l} \times V \times[0, T]\right)$ and $L^{\infty}\left(\Omega_{g} \times V \times[0, T]\right)$. Moreover we have

$$
\begin{aligned}
\left\|f_{\epsilon}\right\|_{\infty}+\left\|g_{\epsilon}\right\|_{\infty} \leq & 2\left\|f_{\epsilon 0}\right\|_{L^{\infty}\left(\Gamma_{1}^{-} \times[0, T]\right)}+2\left\|g_{\epsilon}^{0}\right\|_{L^{\infty}\left(\Omega_{g} \times V\right)}+2\left\|f_{\epsilon}^{0}\right\|_{L^{\infty}\left(\Omega_{l} \times V\right)} \\
& +2\left\|g_{\epsilon 1}\right\|_{L^{\infty}\left(\Gamma_{2}^{-} \times[0, T]\right)}+8 .
\end{aligned}
$$

Finally, we have that $\left.f_{\epsilon}\right|_{\partial \Omega_{l}^{+} \times(0, T)}$ is uniformly bounded in $L^{\infty}\left(\partial \Omega_{l}^{+} \times[0, T]\right)$ and $\left.g_{\epsilon}\right|_{\partial \Omega_{g}^{+} \times(0, T)}$ is uniformly bounded in $L^{\infty}\left(\partial \Omega_{g}^{+} \times[0, T]\right)$.

The proof of this lemma is a direct consequence of the integral representation of the solution given in Theorem 2.1(i) and the assumptions on the initial and boundary data; see also 25 .

Next we present estimates of $f_{\epsilon}, g_{\epsilon}, u_{\epsilon}$ and $w_{\epsilon}$ in $L^{\infty}\left([0, T] ; L^{1}\left(\Omega_{l} \times V\right)\right)$, $L^{\infty}\left([0, T] ; L^{1}\left(\Omega_{g} \times V\right)\right), L^{\infty}\left([0, T] ; L^{1}\left(\Omega_{l}\right)\right)$ and $L^{\infty}\left([0, T] ; L^{1}\left(\Omega_{g}\right)\right)$, respectively.

Lemma 2.2. Assume that

$$
\begin{aligned}
& \left\|f_{\epsilon}^{0}\right\|_{L^{1}\left(\Omega_{l} \times V\right)}<C_{1}, \quad\left\|g_{\epsilon}^{0}\right\|_{L^{1}\left(\Omega_{g} \times V\right)}<C_{2}, \\
& \left\|a(v) \cdot n_{l} f_{\epsilon 0}\right\|_{L^{1}\left(\Gamma_{1}^{-} \times(0, T)\right)}<C_{3}, \quad\left\|a(v) \cdot n_{g} g_{\epsilon 1}\right\|_{L^{1}\left(\Gamma_{2}^{-} \times(0, T)\right)}<C_{4}
\end{aligned}
$$

with $C_{1}, C_{2}, C_{3}$, and $C_{4}$ positive constant independent of $\epsilon$. Then $f_{\epsilon}$ and $g_{\epsilon}$ are uniformly bounded resp. in $L^{\infty}\left([0, T] ; L^{1}\left(\Omega_{l} \times V\right)\right)$ and $L^{\infty}\left([0, T] ; L^{1}\left(\Omega_{g} \times V\right)\right)$, and $u_{\epsilon}$ and $w_{\epsilon}$ are uniformly bounded resp. in $L^{\infty}\left([0, T] ; L^{1}\left(\Omega_{l}\right)\right)$ and $L^{\infty}\left([0, T] ; L^{1}\left(\Omega_{g}\right)\right)$. Moreover, we have

$$
\begin{aligned}
\left\|u_{\epsilon}\right\|_{L^{\infty}\left([0, T] ; L^{1}\left(\Omega_{l}\right)\right)}+\left\|w_{\epsilon}\right\|_{L^{\infty}\left([0, T] ; L^{1}\left(\Omega_{g}\right)\right)} \\
\leq \\
\leq\left\|f_{\epsilon}\right\|_{L^{\infty}\left([0, T] ; L^{1}\left(\Omega_{l} \times V\right)\right)}+\left\|g_{\epsilon}\right\|_{L^{\infty}\left([0, T] ; L^{1}\left(\Omega_{g} \times V\right)\right)} \\
\leq\left\|a(v) \cdot n_{l} f_{\epsilon 0}\right\|_{L^{1}\left(\Gamma_{1}^{-} \times(0, T)\right)}+\left\|a(v) \cdot n_{g} g_{\epsilon 1}\right\|_{L^{1}\left(\Gamma_{2}^{-} \times(0, T)\right)} \\
\quad+\left\|f_{\epsilon}^{0}\right\|_{L^{1}\left(\Omega_{l} \times V\right)}+\left\|g_{\epsilon}^{0}\right\|_{L^{1}\left(\Omega_{g} \times V\right)} .
\end{aligned}
$$


The proof is a direct consequence of formula (15) with $F_{\epsilon} \equiv 0$ and $G_{\epsilon} \equiv 0$.

Next we shall show that under the conditions that the supports in $v \in V$ of the data are compact, the supports in $v \in V$ of the solutions $f_{\epsilon}$ and $g_{\epsilon}$ remain compactly supported with supports included in a fixed compact set independent of $\epsilon$. We shall also give some information about the speed of propagation $a(v)$.

Lemma 2.3. Assume that

$$
\begin{array}{cl}
f_{\epsilon}^{0} \in L^{1}\left(\Omega_{l} \times V\right), & g_{\epsilon}^{0} \in L^{1}\left(\Omega_{g} \times V\right), \\
a(v) \cdot n_{l} f_{\epsilon 0} \in L^{1}\left(\Gamma_{1}^{-} \times(0, T)\right), & a(v) \cdot n_{g} g_{\epsilon 1} \in L^{1}\left(\Gamma_{2}^{-} \times(0, T)\right)
\end{array}
$$

and

$$
\begin{aligned}
& \left\|f_{\epsilon 0}\right\|_{L^{\infty}\left(\Gamma_{1}^{-} \times[0, T]\right)}<C_{1}, \quad\left\|g_{\epsilon}^{0}\right\|_{L^{\infty}\left(\Omega_{g} \times V\right)}<C_{2}, \\
& \left\|f_{\epsilon}^{0}\right\|_{L^{\infty}\left(\Omega_{l} \times V\right)}<C_{3}, \quad\left\|g_{\epsilon 1}\right\|_{L^{\infty}\left(\Gamma_{2}^{-} \times[0, T]\right)}<C_{4}
\end{aligned}
$$

with $C_{1}, C_{2}, C_{3}$, and $C_{4}$ positive constant independent of $\epsilon$. Assume also that the initial and boundary data $f_{\epsilon}^{0}, f_{\epsilon 0}, g_{\epsilon}^{0}$, and $g_{\epsilon 1}$ are compactly supported in $v \in V$ with supports included in a fixed compact set independent of $\epsilon$. Then we have

(i) $u_{\epsilon}$ and $w_{\epsilon}$ are uniformly bounded resp. in $L^{\infty}\left(\Omega_{l} \times[0, T]\right)$ and $L^{\infty}\left(\Omega_{g} \times[0, T]\right)$.

(ii) $f_{\epsilon}$ and $g_{\epsilon}$ remain compactly supported in $v \in V$ with supports included in a fixed compact set independent of $\epsilon$.

(iii) The speed of propagation $a(v)$ is finite.

Proof. (i) Use the integral representation of the solution given in Theorem 2.1] and follow the same lines of the proof of a similar result in the uncoupled case given in [26]. The main difference is the treatment of the contributions to the integral over $\Omega_{l} \times(0, T)$ and $\Omega_{g} \times(0, T)$ involving the interface conditions. This can be handled using the integral representations given in Theorem 2.1 (i). We skip the details.

(ii) Now set $u_{\infty}=\sup _{\epsilon>0}\left\|u_{\epsilon}\right\|_{L^{\infty}\left(\Omega_{l} \times[0, T]\right)}$ and $w_{\infty}=\sup _{\epsilon>0}\left\|w_{\epsilon}\right\|_{L^{\infty}\left(\Omega_{g} \times[0, T]\right)}$. The terms $\chi_{u_{\epsilon}}$ and $\chi_{w_{\epsilon}}$ in the integral representation in Theorem 2.1 are supported by $v \in\left[-u_{\infty}, u_{\infty}\right] \cup\left[-w_{\infty}, w_{\infty}\right]$, the other terms are supported by $v$ in the compact supports of the boundary and initial data. Thus, for all $t \in[0, T], f_{\epsilon}$ and $g_{\epsilon}$ remain compactly supported, with compact supports included in $\operatorname{Supp}_{v} f_{\epsilon}^{0} \cup \operatorname{Supp}_{v} f_{\epsilon 0} \cup$ $\left[-u_{\infty}, u_{\infty}\right]$ resp. $\operatorname{Supp}_{v} g_{\epsilon}^{0} \cup \operatorname{Supp}_{v} g_{\epsilon 1} \cup\left[-w_{\infty}, w_{\infty}\right]$, which in turn are included in a fixed compact set independent of $\epsilon$.

(iii) Set $u_{\infty}=\sup _{\epsilon>0}\left\|u_{\epsilon}\right\|_{L^{\infty}\left(\Omega_{l} \times[0, T]\right)}$ and $w_{\infty}=\sup _{\epsilon>0}\left\|w_{\epsilon}\right\|_{L^{\infty}\left(\Omega_{g} \times[0, T]\right)}$. By (i) above, these definitions make sense. Let $S=\operatorname{supp}_{v} f_{\epsilon}^{0} \cup \operatorname{supp}_{v} f_{\epsilon 0} \cup\left[-u_{\infty}, u_{\infty}\right] \cup$ $\operatorname{supp}_{v} g_{\epsilon}^{0} \cup \operatorname{supp}_{v} g_{\epsilon 1} \cup\left[-w_{\infty}, w_{\infty}\right]$. Then the support in $v$ of $f_{\epsilon}$ resp. $g_{\epsilon}$ is included in $S$. Now set $a_{\infty}=\sup _{1 \leq i \leq N, v \in S}\left|a_{i}(v)\right|$. We conclude that $\sup _{1 \leq i \leq N, v \in S_{11} \cup S_{12}}\left|a_{i}(v)\right|$ $\leq a_{\infty}$, where $S_{11}=\left\{v \in \operatorname{supp}_{v} f_{\epsilon}(x, ., t),(x, t) \in \Omega_{l} \times(0, \bar{T})\right\}$ and $S_{12}=\{v \in$ $\left.\operatorname{supp}_{v} g_{\epsilon}(x, ., t),(x, t) \in \Omega_{g} \times(0, T)\right\}$. The lemma is proved.

2.4. Gauss-Green formula. Let $X$ be an open set of $\mathbb{R}^{d}$. Assume that

$$
\Gamma_{0}=\{(x, v) \in \partial X \times V: a(v) \cdot n(x)=0\}
$$

has measure 0 in $\partial X \times V$ for the measure $d \gamma d v$. Recall from the introduction that this condition is satisfied for $\Gamma_{0 l}$ and $\Gamma_{0 g}$.

Let $W^{p}$ denote the space

$$
W^{p}=\left\{u \in L^{p}(X \times V \times(0, T)) ;\left(\partial_{t}+a(v) \cdot \partial_{x}\right) u \in L^{p}(X \times V \times(0, T))\right\},
$$

where $1 \leq p<\infty$. Then we have the following theorem. 
Theorem 2.3. Let $K$ be a compact set of $(\partial X)^{-} \times(0, T)\left(\right.$ resp. $\left.(\partial X)^{+} \times(0, T)\right)$. Then the mapping

$$
u \rightarrow u \mid K,
$$

defined on $\mathcal{D}(\bar{X} \times V \times(0, T))$, can be extended to a continuous mapping from $W^{p}$ to $L^{p}(K)$. As a consequence the functions of $W^{p}(p \in[1,+\infty))$ have a trace in $L_{l o c}^{p}\left((\partial X)^{-} \times(0, T)\right)$ (resp. $L_{l o c}^{p}\left((\partial X)^{+} \times(0, T)\right)$. Here $(\partial X)^{-}$and $(\partial X)^{+}$are defined according to the definition of $\Gamma^{-}$and $\Gamma^{+}$given in the Introduction.

When $a(v)=v$, this result has been proven in [6]. The proof for the case of general $a(v)$ satisfying the assumptions made at the beginning of this paper and above, is based on a slight modification of the proof for the case $a(v)=v$. As a consequence of the above theorem, we have the Green's formula

Theorem 2.4. Let $f \in W^{1}$. Then $\forall \psi \in C_{0}^{1}(\bar{X} \times V \times(0, T))$ we have

$$
\begin{gathered}
\int_{X \times V \times(0, T)}\left[\partial_{t}+a(v) \cdot \partial_{x}\right] f \psi+\int_{X \times V \times(0, T)} f\left[\partial_{t}+a(v) \cdot \partial_{x}\right] \psi \\
(25)=\int_{(\partial X)+\times(0, T)} a(v) \cdot n(x) f \psi d \gamma d v d t+\int_{(\partial X)-\times(0, T)} a(v) \cdot n(x) f \psi d \gamma d v d t .
\end{gathered}
$$

Now let $U \subset \mathbb{R}^{N}$ be an open bounded subset whose boundary $\partial U$ is regular Lipschitz deformable [4]. That is, we have

(i) $\forall u \in \partial U, \exists r>0$ and a Lipschitz map $\gamma: \mathbb{R}^{N-1} \rightarrow \mathbb{R}$ such that after rotating and relabling coordinates if necessary,

$$
U \cap Q(u, r)=\left\{y \in \mathbb{R}^{N}: \gamma\left(y_{1}, \cdots, y_{N-1}\right)<y_{N}\right\} \cap Q(u, r),
$$

where $Q(u, r)=\left\{y \in \mathbb{R}^{N}:\left|u_{i}-y_{i}\right| \leq r, i=1, \cdots, N\right\}$.

(ii) $\exists \Psi: \partial U \times[0,1] \rightarrow \bar{U}$ such that $\Psi$ is a homeomorphism bi-Lipschitz over its image and $\Psi(u, 0)=u, \forall u \in \partial U$.

(iii) Let $\partial U_{z} \equiv \Psi(\partial U \times\{z\}), z \in[0,1]$ and let $U_{z}$ be the open subset of $U$ whose boundary is $\partial U_{z}$ and $\Psi$ is as in (ii). Let $\Psi_{z}: \partial U \rightarrow \bar{U}$ be such that $\Psi_{z}(u)=\Psi(u, z)$ for any $(u, z) \in \partial U \times[0,1] . \Psi_{z}$ and $\gamma$ satisfy

$$
\operatorname{limit}_{z \rightarrow 0^{+}} D \Psi_{z} \circ \gamma=D \gamma \quad \text { in } L_{l o c}^{1}(\mathcal{O}),
$$

where $\mathcal{O}$ denotes the greatest open set such that $\gamma(\mathcal{O}) \subset \partial U$.

When $\partial U$ is such that (i) and (ii) are satisfied, we say that $\partial U$ is Lipschitz deformable and $\Psi$ is a Lipschitz deformation of $\partial U$. If a Lipschitz deformable boundary $\partial U$ satisfies (iii), then the Lipschitz deformation $\Psi$ of $\partial U$ is called regular. Examples of domains whose boundaries are regular Lipschitz deformable include bounded domains with smooth $\left(C^{2}\right.$ for example) boundaries; star-shaped domains and those satisfying the cone property; see 4 .

Let $\nu: \Psi(\partial U \times[0,1]) \rightarrow \mathbb{R}^{N}$ be such that $\nu(u)$ is the unit outward normal to $\partial U_{z}$ at $u \in \partial U_{z} \equiv \Psi(\partial U \times\{z\})$ defined a.e. We have [4]

Theorem 2.5. Let $F$ be a function in $L^{\infty}\left(U ; \mathbb{R}^{N}\right)$ whose divergence is a bounded measure. Then there exists a continuous linear functional $\left.F \cdot \nu\right|_{\partial U}$ over $\operatorname{Lip}(\partial U)$ such that

$$
\left\langle\left. F \cdot \nu\right|_{\partial U}, \phi\right\rangle=\int_{U} \phi \operatorname{div} F+\int_{U} \nabla \phi \cdot F \quad \forall \phi \in \operatorname{Lip}\left(\mathbb{R}^{N}\right) .
$$

Moreover, the normal trace $\left.F \cdot \nu\right|_{\partial U}$ is a function in $L^{\infty}(\partial U)$ satisfying

$$
\|F \cdot \nu\|_{L^{\infty}(\partial U)} \leq C\|F\|_{L^{\infty}(U)}
$$


for some constant $C$ independent of $F$ and

$$
\left\langle F \cdot \nu_{\mid \partial U}, \psi\right\rangle=\text { ess } \text { limit }_{z \rightarrow 0} \int_{\partial U_{z}} \psi \circ \Psi_{z}^{-1} F \cdot \nu d \mathcal{H}^{N-1} \quad \forall \psi \in \operatorname{Lip}(\partial U) .
$$

\section{Kinetic FORMUlation OF THE COUPLED KINETIC-HYDRODYNAMIC SYSTEM}

We begin by introducing a definition for a physically correct solution to problem (8) $-(13)$.

Definition 3.1. Let $f \in L^{\infty}\left([0, T] ; L^{1}\left(\Omega_{l} \times V\right)\right) \cap L^{\infty}\left(\Omega_{l} \times[0, T] ; L^{1}(V)\right)$ and $w \in$ $L^{\infty}\left(\Omega_{g} \times(0, T)\right) \cap L^{\infty}\left([0, T] ; L^{1}\left(\Omega_{g}\right)\right)$. We say that $(f, w)$ is a weak entropy solution of system (8)-(13) if the equations (8) and (11) are satisfied in the distribution sense, the weak traces of $f$ on $\Gamma_{1}^{+} \times(0, T)$ and $\Gamma_{i}^{+} \times(0, T)$ and $\chi_{w}$ on $\Gamma_{i}^{-} \times(0, T)$ exist, and the following holds:

$$
\begin{aligned}
& -\int_{\Omega_{l} \times V \times(0, T)}\left[\partial_{t}+a(v) \cdot \partial_{x}\right]\left(\psi_{1}\right) f+\int_{\Gamma_{1}^{-} \times(0, T)} a(v) \cdot n_{l} \psi_{1} f_{0} \\
& +\int_{\Gamma_{1}^{+} \times(0, T)} a(v) \cdot n_{l} \psi_{1} f+\int_{\Gamma_{i}^{-} \times(0, T)} a(v) \cdot n_{l} \psi_{1} \chi_{w}+\int_{\Gamma_{i}^{+} \times(0, T)} a(v) \cdot n_{l} \psi_{1} f \\
& =\frac{1}{\epsilon_{l}} \int_{\Omega_{l} \times V \times(0, T)}\left(\chi_{u(x, t)}(v)-f(x, v, t)\right)\left(\psi_{1}\right)
\end{aligned}
$$

and

$$
\begin{aligned}
& -\int_{\Omega_{g} \times(0, T)}\left(|w-k| \partial_{t} \psi_{2}+\operatorname{sign}(w-k)(A(w)-A(k)) \cdot \nabla_{x} \psi_{2}\right) \\
& +\int_{\Gamma_{2} \times(0, T)} \psi_{2} \operatorname{sign}\left(w_{1}-k\right)\left(\left(A\left(w_{1}\right) \cdot n_{g}\right)^{-}-(A(k) \cdot n)^{-}\right) \\
& +\int_{\Gamma_{i}^{+} \times(0, T)} a(v) \cdot n_{g} \psi_{2}\left|f-\chi_{k}\right| \leq 0 \\
& \forall \psi_{1} \in C_{0}^{1}\left(\bar{\Omega}_{l} \times V \times(0, T)\right), \quad \forall \psi_{2} \in C_{0}^{1}\left(\bar{\Omega}_{g} \times(0, T)\right), \psi_{2} \geq 0, \quad \forall k \in \mathbb{R}
\end{aligned}
$$

and the initial conditions

$$
f(x, v, 0)=f^{0}(x, v) \text { in } \Omega_{l} \times V \quad w(x, 0)=w^{0}(x) \text { in } \Omega_{g} .
$$

Remark 3.1. (i) Since by definition $f \in L^{\infty}\left([0, T] ; L^{1}\left(\Omega_{l} \times V\right)\right) \cap L^{\infty}\left(\Omega_{l} \times[0, T] ; L^{1}(V)\right)$, $f \in L^{1}\left(\Omega_{l} \times(0, T) \times L^{1}(V)\right)$ and $\left(\partial_{t}+a(v) \cdot \partial_{x}\right) f \in L^{1}\left(\Omega_{l} \times V \times(0, T)\right)$ (see the equation satisfied by $f$ ). Thus, we can apply Theorems 2.3 and 2.4 Therefore, the trace of $f$ on $\left(\partial \Omega_{l}\right)^{+} \times(0, T)$ is in $L_{l o c}^{1}\left(\left(\partial \Omega_{l}\right)^{+} \times(0, T)\right)$. This justifies the integrals involving the boundary terms of $f$. The existence of the weak trace of $\chi_{w}$ is proved in Theorem 4.10 which is given at the end of the paper. For more details consult the proof of Theorem 4.8 .

(ii) Below we shall make precise the boundary conditions for $w$ on $\partial \Omega_{g} \times(0, T)$. Taking $\psi_{2} \in C_{0}^{1}\left(\Omega_{g} \times(0, T)\right)$ in the definition above, we obtain

(29) $\partial_{t}|w-k|+\operatorname{div}[\operatorname{sgn}(w-k)(A(w)-A(k))] \leq 0$ in $\mathcal{D}^{\prime}\left(\Omega_{g} \times(0, T)\right)$.

Therefore $\partial_{t}|w-k|+\operatorname{div}[\operatorname{sgn}(w-k)(A(w)-A(k))]$ is a bounded measure on $\Omega_{g} \times(0, T)$. Set $F=(|w-k|, \operatorname{sgn}(w-k)(A(w)-A(k)))$; then it is clear that $F \in L^{\infty}\left(\Omega_{g} \times(0, T) ; \mathbb{R}^{d+1}\right)$. On the other hand, using the above,

$$
\operatorname{div}_{t, x} F=\partial_{t}|w-k|+\operatorname{div}[\operatorname{sgn}(w-k)(A(w)-A(k))]
$$


is a bounded measure on $U=\Omega_{g} \times(0, T)$. Hence we can use Theorem 2.5 which yields

$$
\begin{aligned}
& \text { ess } \operatorname{limit}_{z \rightarrow 0} \int_{\partial U_{z}} \phi \circ \Psi_{z}^{-1} F \cdot \nu d \mathcal{H}^{d} \\
& =\int_{\Omega_{g} \times(0, T)} \phi\left(\partial_{t}|w-k|+\operatorname{div}[\operatorname{sgn}(w-k)(A(w)-A(k))]\right)
\end{aligned}
$$

$$
+\int_{\Omega_{g} \times(0, T)}\left(|w-k| \partial_{t} \phi+\operatorname{sgn}(w-k)(A(w)-A(k)) \cdot \nabla_{x} \phi\right) \quad \forall \phi \in \operatorname{Lip}\left(\mathbb{R}^{d+1}\right),
$$

where $\nu=\left(\nu_{1}, \nu_{\star}\right)$ and $\Psi$ were introduced in the previous section. Taking $\psi_{2}=\phi \in$ $C_{0}^{1}\left(\bar{\Omega}_{g} \times(0, T)\right), \psi_{2} \geq 0$, in Definition 3.1 and using (29) and (30), we obtain

$$
\begin{aligned}
& -\operatorname{ess~limit~}_{z \rightarrow 0} \int_{\partial \Omega_{g} \times(0, T)} \phi \circ \Psi_{z}^{-1} \operatorname{sgn}(w-k)(A(w)-A(k)) \cdot \nu_{\star} d \mathcal{H}^{d} \\
& +\int_{\Omega_{g} \times(0, T)} \phi\left(\partial_{t}|w-k|+\operatorname{div}[\operatorname{sgn}(w-k)(A(w)-A(k))]\right) \\
& +\int_{\Gamma_{2} \times(0, T)} \psi_{2} \operatorname{sign}\left(w_{1}-k\right)\left(\left(A\left(w_{1}\right) \cdot n_{g}\right)^{-}-\left(A(k) \cdot n_{g}\right)^{-}\right) \\
& +\int_{\Gamma_{i}^{+} \times(0, T)} a(v) \cdot n_{g} \psi_{2}\left|f-\chi_{k}\right| \leq 0 .
\end{aligned}
$$

For $\psi_{2} \in C_{0}^{1}\left(\partial \Omega_{g} \times(0, T)\right)$, this yields

$$
\begin{aligned}
& -\operatorname{ess~limit~}_{z \rightarrow 0} \int_{\partial \Omega_{g} \times(0, T)} \phi \circ \Psi_{z}^{-1} \operatorname{sgn}(w-k)(A(w)-A(k)) \cdot \nu_{\star} d \mathcal{H}^{d} \\
& +\int_{\Gamma_{2} \times(0, T)} \psi_{2} \operatorname{sign}\left(w_{1}-k\right)\left(\left(A\left(w_{1}\right) \cdot n_{g}\right)^{-}-\left(A(k) \cdot n_{g}\right)^{-}\right) \\
& +\int_{\Gamma_{i}^{+} \times(0, T)} a(v) \cdot n_{g} \psi_{2}\left|f-\chi_{k}\right| \leq 0 .
\end{aligned}
$$

This in turn yields

$$
\begin{aligned}
& - \text { ess limit }_{z \rightarrow 0} \int_{\Psi\left(\Gamma_{2} \times\{z\}\right) \times(0, T)} \psi_{2} \circ \Psi_{z}^{-1} \operatorname{sgn}(w-k)(A(w)-A(k)) \cdot \nu_{\star} d \mathcal{H}^{d} \\
& +\int_{\Gamma_{2} \times(0, T)} \psi_{2} \operatorname{sign}\left(w_{1}-k\right)\left(\left(A\left(w_{1}\right) \cdot n_{g}\right)^{-}-\left(A(k) \cdot n_{g}\right)^{-}\right) \leq 0 \\
& \forall \psi_{2} \in C_{0}^{1}\left(\Gamma_{2} \times(0, T)\right)
\end{aligned}
$$

and

$$
\begin{aligned}
& -\operatorname{ess~}_{\operatorname{limit}}^{z \rightarrow 0} \int_{\Psi\left(\Gamma_{i} \times\{z\}\right) \times(0, T)} \psi_{2} \circ \Psi_{z}^{-1} \operatorname{sgn}(w-k)(A(w)-A(k)) \cdot \nu_{\star} d \mathcal{H}^{d} \\
& +\int_{\Gamma_{i}^{+} \times(0, T)} a(v) \cdot n_{g} \psi_{2}\left|f-\chi_{k}\right| \leq 0 \\
& \forall \psi_{2} \in C_{0}^{1}\left(\Gamma_{i} \times(0, T)\right) .
\end{aligned}
$$

This clarifies the boundary conditions for $w$. 
Let $f_{l}(x, v, t)$ and $g(x, v, t)$ be functions defined respectively in $\Omega_{l} \times V \times(0, T)$ and $\Omega_{g} \times V \times(0, T)$. We introduce the following system:

$$
\begin{aligned}
& {\left[\partial_{t}+a(v) \cdot \partial_{x}\right] f_{l}(x, v, t)=\frac{1}{\epsilon_{l}}\left(\chi_{u_{l}(x, t)}(v)-f_{l}(x, v, t)\right) \text { in } \Omega_{l} \times V \times(0, T)} \\
& f_{l}(x, v, t)=f_{0}(x, v, t) \text { on } \Gamma_{1}^{-} \times(0, T), \\
& f_{l}(x, v, t)=g(x, v, t) \text { on } \Gamma_{i}^{-} \times(0, T) \\
& {\left[\partial_{t}+a(v) \cdot \partial_{x}\right] g(x, v, t)=\frac{\partial m}{\partial v} \text { in } \Omega_{g} \times V \times(0, T)} \\
& g(x, v, t)=\chi_{w(x, t)}(v) \text { in } \Omega_{g} \times V \times(0, T), \\
& g(x, v, t)=\chi_{w_{1}(x, t)}(v) \text { on } \Gamma_{2}^{-} \times(0, T), \\
& g(x, v, t)=f_{l}(x, v, t) \text { on } \Gamma_{i}^{+} \times(0, T), \\
& m \text { nonnegative bounded measure in } \Omega_{g} \times V \times(0, T), \\
& f_{l}(x, v, 0)=f^{0}(x, v) \text { in } \Omega_{l} \times V \quad g(x, v, 0)=g^{0}(x, v) \text { in } \Omega_{g} \times V,
\end{aligned}
$$

where $f_{0}, w_{1}$, and $f^{0}$ were introduced in Section 1 . The function $u_{l}$ is related to $f_{l}$ by $u_{l}=\int_{V} f_{l}$. The function $g^{0}$ is related to $w^{0}$ by

$$
g^{0}(x, v)=\chi_{w^{0}(x)}(v) \text { in } \Omega_{g} \times V .
$$

The boundary conditions in (35), (36), (39), and (40) will be made precise in the following definition of a solution to the above system.

Definition 3.2. We say that

$$
\begin{aligned}
\left(f_{l}, g\right) & \in\left(L^{\infty}\left([0, T] ; L^{1}\left(\Omega_{l} \times V\right)\right) \cap L^{\infty}\left(\Omega_{l} \times[0, T] ; L^{1}(V)\right)\right) \\
& \times\left(L^{\infty}\left([0, T] ; L^{1}\left(\Omega_{g} \times V\right)\right) \cap L^{\infty}\left(\Omega_{g} \times(0, T) ; L^{1}(V)\right)\right)
\end{aligned}
$$

is a weak solution of the system (34)-(42) if the equations (34) and (37) are satisfied in the distribution sense, the weak traces of $f_{l}$ on $\Gamma_{1}^{+} \times(0, T)$ and on $\Gamma_{i}^{+} \times(0, T)$ and $g$ on $\Gamma_{i}^{-} \times(0, T)$ exist, and we have

$$
\begin{aligned}
& -\int_{\Omega_{l} \times V \times(0, T)}\left(\partial_{t}+a(v) \cdot \partial_{x}\right)\left(\psi_{1}\right) f_{l}+\int_{\Gamma_{1}^{-} \times(0, T)} a(v) \cdot n \psi_{1} f_{0} \\
& +\int_{\Gamma_{1}^{+} \times(0, T)} a(v) \cdot n \psi_{1} f_{l}+\int_{\Gamma_{i}^{-} \times(0, T)} a(v) \cdot n \psi_{1} g \\
& +\int_{\Gamma_{i}^{+} \times(0, T)} a(v) \cdot n \psi_{1} f_{l}=\frac{1}{\epsilon_{l}} \int_{\Omega_{l} \times V \times(0, T)}\left(\chi_{u_{l}(x, t)}(v)-f_{l}(x, v, t)\right)\left(\psi_{1}\right)
\end{aligned}
$$

and

$$
\begin{aligned}
& -\int_{\Omega_{g} \times V \times(0, T)}\left(\partial_{t}+a(v) \cdot \partial_{x}\right)\left(\psi_{2}\right)\left|g-\chi_{k}\right|+\int_{\Gamma_{2}^{-} \times(0, T)} a(v) \cdot n \psi_{2}\left|\chi_{w_{1}}-\chi_{k}\right| \\
& +\int_{\Gamma_{i}^{+} \times(0, T)} a(v) \cdot n \psi_{2}\left|f_{l}-\chi_{k}\right| \leq 0 \\
& \forall \psi_{1} \in C_{0}^{1}\left(\bar{\Omega}_{l} \times V \times(0, T)\right), \quad \forall \psi_{2} \in C_{0}^{1}\left(\bar{\Omega}_{g} \times(0, T)\right), \quad \psi_{2} \geq 0, \forall k \in \mathbb{R}
\end{aligned}
$$

and the initial conditions

$$
f_{l}(x, v, 0)=f^{0}(x, v) \text { in } \Omega_{l} \times V \quad g(x, v, 0)=g^{0}(x, v) \text { in } \Omega_{g} \times V .
$$


Remark 3.2. Notice that Remark 3.1 applies here also.

The following theorem states the equivalence between the two systems (8)-(13) and (34)-(42).

Theorem 3.1. (i) Let $f \in L^{\infty}\left([0, T] ; L^{1}\left(\Omega_{l} \times V\right)\right) \cap L^{\infty}\left(\Omega_{l} \times[0, T] ; L^{1}(V)\right)$ and $w \in L^{\infty}\left(\Omega_{g} \times[0, T]\right) \cap L^{\infty}\left([0, T] ; L^{1}\left(\Omega_{g}\right)\right)$ solve (8)-(13) in the sense of Definition 3.1. Set $f_{l}=f$ and $g(x, v, t)=\chi_{w(x, t)}(v)$. Then $f_{l} \in L^{\infty}\left([0, T] ; L^{1}\left(\Omega_{l} \times V\right)\right) \cap$ $L^{\infty}\left(\Omega_{l} \times[0, T] ; L^{1}(V)\right)$ and $g \in L^{\infty}\left([0, T] ; L^{1}\left(\Omega_{g} \times V\right)\right) \cap L^{\infty}\left(\Omega_{g} \times[0, T] ; L^{1}(V)\right)$ solve in the sense of Definition 3.2 the system (34)-(142) for some $m$ supported in $\Omega_{g} \times\left[-R_{g}, R_{g}\right] \times(0, T)$ with $R_{g}=\|w\|_{L^{\infty}}$.

(ii) Let $f_{l} \in L^{\infty}\left([0, T] ; L^{1}\left(\Omega_{l} \times V\right)\right) \cap L^{\infty}\left(\Omega_{l} \times[0, T] ; L^{1}(V)\right)$ and $g \in L^{\infty}([0, T]$; $\left.L^{1}\left(\Omega_{g} \times V\right)\right) \cap L^{\infty}\left(\Omega_{g} \times[0, T] ; L^{1}(V)\right)$ solve (34) -(42). Set $w(x, t)=\int_{V} g(x, v, t) d v$ and $f=f_{l}$. Then $w \in L^{\infty}\left(\Omega_{g} \times[0, T]\right) \cap L^{\infty}\left([0, T] ; L^{1}\left(\Omega_{g}\right)\right)$ and $(f, w)$ solves (8) -(13) in the sense of Definition 3.1.

Remark 3.3. The kinetic formulation of the coupled system (8)-(13) given above is inspired from the kinetic formulation of conservation laws with boundaries introduced by the author in [27], which is in turn based on the generalization of kinetic formulation introduced in [12] to conservation laws for domains with boundaries.

Proof of Theorem 3.1. We begin with the proof of (i). The construction of $m$, the proof of its boundedness, and the proof that $g$ and $m$ satisfy Eq. (37) are similar to those given in 12. Therefore we refer to that paper and give detail only for the new aspects of the formulation, which are related to the boundary and transmission conditions.

Using Eq. (26) in Definition 3.1 we conclude that $\left(f_{l}, g\right)$ satisfies the first part of Definition 3.2 i.e. Eq. (44). Now using Eq. (27) and the properties of $\chi$, we conclude that $\left(f_{l}, g\right)$ satisfies the second part of Definition 3.2, i.e. Eq. (45). Finally, since $(f, w)$ satisfies the initial condition (28), using (43) and the properties of $\chi,\left(f_{l}, w\right)$ satisfies the initial condition (46).

We now prove the second part of the theorem. Eq. (8) is clearly satisfied. On the other hand

$$
\left\langle\frac{\partial g}{\partial t}+\sum_{i=1}^{d} a_{i}(v) \cdot \frac{\partial g}{\partial x_{i}}, h \otimes 1\right\rangle=0
$$

$\forall h \in \mathcal{D}\left(\Omega_{g} \times(0, T)\right.$. Using the properties of $\chi$, we conclude that Eq. (11) is satisfied in the distribution sense.

We now wish to prove that the remaining requirements in Definition 3.1 are satisfied. First, using Eq. (44), Eq. (26) is satisfied. To prove that Eq. (27) is satisfied, we just use Eq. (45) and the properties of $\chi$. Finally, since $\left(f_{l}, g\right)$ satisfies the initial condition (46), using (43) and the properties of $\chi,(f, w)$ satisfies the initial condition (28). The theorem is now proved.

\section{Analysis of the KinetiC FORMUlation}

In this section we shall study the behavior of system (1)-(17), as the microscopic scale in $\Omega_{g}$ tends to 0 . We prove that as $\epsilon \rightarrow 0$, the solution of the coupled kinetic system converges to a solution of system (34)-(42). The existence theory for the system (8)-(13) will be deduced from Theorem 3.1 using the kinetic formulation of the coupled system. 
In the sequel, we shall assume that $\Omega_{l}=(0, l)$ and $\Omega_{g}=(l, 1)$. It is not difficult to generalize our proof to the case $\Omega_{l}=(0, l) \times \mathbb{R}^{d-1}$ and $\Omega_{g}=(l, 1) \times \mathbb{R}^{d-1}$. Throughout the rest of this paper the terminology convergence of $h_{\epsilon}$ as $\epsilon \rightarrow 0$ means convergence up to a subsequence of $h_{\epsilon}$, still denoted $h_{\epsilon}$, and does not necessarily mean convergence of the whole sequence. Also ess $\lim _{z \rightarrow 0}$ will simply be denoted by $\lim _{z \rightarrow 0}$.

We first introduce a nondegeneracy assumption on the curve: $v \rightarrow a(v)$ in $\mathbb{R}^{d}$. This relation is needed in order to use regularity results for the averages. Assume that the initial and boundary data satisfy

$$
\begin{aligned}
& \left\|f_{\epsilon}^{0}\right\|_{L^{1}\left(\Omega_{l} \times V\right)}<C_{1}, \quad\left\|g_{\epsilon}^{0}\right\|_{L^{1}\left(\Omega_{g} \times V\right)}<C_{2}, \\
& \left\|a(v) \cdot n_{l} f_{\epsilon 0}\right\|_{L^{1}\left(\Gamma_{1}^{-} \times(0, T)\right)}<C_{3},\left\|a(v) \cdot n_{g} g_{\epsilon 1}\right\|_{L^{1}\left(\Gamma_{2}^{-} \times(0, T)\right)}<C_{4}, \\
& \left\|f_{\epsilon 0}\right\|_{L^{\infty}\left(\Gamma_{1}^{-} \times[0, T]\right)}<C_{5},\left\|g_{\epsilon}^{0}\right\|_{L^{\infty}\left(\Omega_{g} \times V\right)}<C_{6}, \\
& \left\|f_{\epsilon}^{0}\right\|_{L^{\infty}\left(\Omega_{l} \times V\right)}<C_{7}, \quad\left\|g_{\epsilon 1}\right\|_{L^{\infty}\left(\Gamma_{2}^{-} \times[0, T]\right)}<C_{8}
\end{aligned}
$$

with $C_{1}, \cdots, C_{8}$ positive constants independent of $\epsilon$. Assume also that the initial and boundary data $f_{\epsilon}^{0}, f_{\epsilon 0}, g_{\epsilon}^{0}$, and $g_{\epsilon 1}$ are compactly supported in $v \in V$ with supports included in a fixed compact set independent of $\epsilon$. Then by Lemma 2.2 $u_{\epsilon}$ resp. $w_{\epsilon}$ is uniformly bounded in $L^{\infty}\left([0, T] ; L^{1}\left(\Omega_{l}\right)\right)$ resp. $L^{\infty}\left([0, T] ; L^{1}\left(\Omega_{g}\right)\right)$, and by Lemma $2.3 u_{\epsilon}$ resp. $w_{\epsilon}$ is uniformly bounded in $L^{\infty}\left(\Omega_{l} \times[0, T]\right)$ resp. in $L^{\infty}\left(\Omega_{g} \times[0, T]\right)$. Let $R_{\infty}$ denote the common bound of $u_{\epsilon}$ in $L^{\infty}\left([0, T] ; L^{1}\left(\Omega_{l}\right)\right)$ and $L^{\infty}\left(\Omega_{l} \times[0, T]\right)$ and of $w_{\epsilon}$ in $L^{\infty}\left([0, T] ; L^{1}\left(\Omega_{g}\right)\right)$ and $L^{\infty}\left(\Omega_{g} \times[0, T]\right)$. We then make the following assumption on the curve $a(\cdot)$ :

(48) meas $\left\{|v|<R_{\infty}, \tau+a(v) \cdot \xi=0\right\}=0 \quad \forall(\tau, \xi) \in \mathbb{R} \times \Omega_{g}$ such that $\tau^{2}+\xi^{2}=1$.

This condition was introduced in 12 . In the case $d=1$ this relation is actually stronger than a related condition introduced by Tartar [18]. For the description of examples of fluxes $A$ satisfying (48) and examples of fluxes $A$ where this condition fails, we refer to [12].

We begin with a compactness result for $u_{\epsilon}$.

Theorem 4.1. Assume that

$$
\begin{aligned}
& \left\|f_{\epsilon 0}\right\|_{L^{\infty}\left(\Gamma_{1}^{-} \times[0, T]\right)}<C_{1}, \quad\left\|g_{\epsilon}^{0}\right\|_{L^{\infty}\left(\Omega_{g} \times V\right)}<C_{2}, \quad\left\|f_{\epsilon}^{0}\right\|_{L^{\infty}\left(\Omega_{l} \times V\right)}<C_{3}, \\
& \left\|g_{\epsilon 1}\right\|_{L^{\infty}\left(\Gamma_{2}^{-} \times[0, T]\right)}<C_{4},\left\|f_{\epsilon}^{0}\right\|_{L^{1}\left(\Omega_{l} \times V\right)}<C_{5},\left\|g_{\epsilon}^{0}\right\|_{L^{1}\left(\Omega_{g} \times V\right)}<C_{6}, \\
& \left\|a(v) \cdot n_{l} f_{\epsilon 0}\right\|_{L^{1}\left(\Gamma_{1}^{-} \times(0, T)\right)}<C_{7},\left\|a(v) \cdot n_{g} g_{\epsilon 1}\right\|_{L^{1}\left(\Gamma_{2}^{-} \times(0, T)\right)}<C_{8}
\end{aligned}
$$

with $C_{i}, i=1, \cdots, 8$, positive constants independent of $\epsilon$.

Assume also that the initial and boundary data $f_{\epsilon}^{0}, f_{\epsilon 0}, g_{\epsilon}^{0}$, and $g_{\epsilon 1}$ are compactly supported in $v \in V$ with supports included in a fixed compact set independent of $\epsilon$. Finally assume that (48) hold. Then a subsequence of $u_{\epsilon}=\int_{\mathbb{R}} f_{\epsilon} d v$ (still denoted $\left.u_{\epsilon}\right)$ converges strongly in $L^{1}\left(\Omega_{l} \times(0, T)\right)$ to a function $u \in L^{1}\left(\Omega_{l} \times(0, T)\right)$.

The proof of this statement is based on the following averaging lemma.

Theorem 4.2. Let $f \in L^{q}\left(\Omega_{l} \times V \times(0, T)\right)$, let

$$
\partial f / \partial t+a(v) \cdot \nabla_{x} f \in L^{q}\left(\Omega_{l} \times V \times(0, T)\right), q>1,
$$

let $\left.f\right|_{t=0}=f^{0} \in L^{\infty}\left(\Omega_{l} \times V\right)$, and let $\left.f\right|_{\left(\partial \Omega_{l}\right)^{-}}=f_{b} \in L^{\infty}\left(\left(\partial \Omega_{l}\right)^{-} \times(0, T)\right)$. Assume also that (48) is satisfied. 
Then $\int_{V} f d v \in W^{s, q}\left(\Omega_{l} \times(0, T)\right)$ for any $s, 0<s<\inf (1 / q, 1-1 / q)$, and

$$
\left\|\int_{V} f d v\right\|_{W^{s, q}} \leq C\left(f_{b}, f^{0}\right)\left(\|f\|_{L^{q}}+\left\|\partial f / \partial t+a(v) \cdot \nabla_{x} f\right\|_{L^{q}}\right) .
$$

When $a(v)=v$ Theorem 4.2 is exactly the same as the averaging lemma given in [9 1] and proved in [9]. Therefore our lemma is a slight generalization of the lemma in [9, 1]. The proof is similar to the proof given in [9].

Proof of Theorem 4.1. First, we observe that Lemmas 2.2 2.3 show that $f_{\epsilon}$ and $\partial f_{\epsilon} / \partial t+a(v) \cdot \nabla_{x} f_{\epsilon}$ are uniformly bounded in $L^{q}(\Omega \times V \times(0, T))$ for any $q \geq 1$. Lemma 2.1, together with the transmission condition (3) and the integral representation in Theorem 2.1, shows that $\left.f_{\epsilon}\right|_{\Gamma_{i}^{-}}$is uniformly bounded in $L^{\infty}\left(\Gamma_{i}^{-} \times(0, T)\right)$. Moreover, we know by assumption that $f_{\epsilon 0}$ resp. $f_{\epsilon}^{0}$ is uniformly bounded in $L^{\infty}\left(\Gamma_{1}^{-} \times(0, T)\right)$ resp. $L^{\infty}\left(\Omega_{l} \times V\right)$. Therefore we can use Theorem 4.2 and the uniform boundedness of $u_{\epsilon}$ in $L^{\infty}\left(\Omega_{l} \times(0, T)\right)$ to infer that a subsequence of $u_{\epsilon}$ (still denoted $u_{\epsilon}$ ) converges in $L^{1}\left(\Omega_{l} \times(0, T)\right)$ to a function $u \in L^{1}\left(\Omega_{l} \times(0, T)\right.$ ). This concludes the proof of the theorem.

Theorem 4.3. Assume that

$$
\begin{aligned}
& \left\|f_{\epsilon 0}\right\|_{L^{\infty}\left(\Gamma_{1}^{-} \times[0, T]\right)}<C_{1}, \quad\left\|g_{\epsilon}^{0}\right\|_{L^{\infty}\left(\Omega_{g} \times V\right)}<C_{2}, \quad\left\|f_{\epsilon}^{0}\right\|_{L^{\infty}\left(\Omega_{l} \times V\right)}<C_{3}, \\
& \left\|g_{\epsilon 1}\right\|_{L^{\infty}\left(\Gamma_{2}^{-} \times[0, T]\right)}<C_{4},\left\|f_{\epsilon}^{0}\right\|_{L^{1}\left(\Omega_{l} \times V\right)}<C_{5},\left\|g_{\epsilon}^{0}\right\|_{L^{1}\left(\Omega_{g} \times V\right)}<C_{6}, \\
& \left\|a(v) \cdot n_{l} f_{\epsilon 0}\right\|_{L^{1}\left(\Gamma_{1}^{-} \times(0, T)\right)}<C_{7}, \quad\left\|a(v) \cdot n_{g} g_{\epsilon 1}\right\|_{L^{1}\left(\Gamma_{2}^{-} \times(0, T)\right)}<C_{8}
\end{aligned}
$$

with $C_{i}, i=1, \cdots, 8$, positive constants independent of $\epsilon$.

Assume also that the initial and boundary data $f_{\epsilon}^{0}, f_{\epsilon 0}, g_{\epsilon}^{0}$, and $g_{\epsilon 1}$ are compactly supported in $v \in V$ with supports included in a fixed compact set independent of $\epsilon$. Finally assume that (48) holds. Then:

(i) A subsequence of $f_{\epsilon}$ (still denoted $f_{\epsilon}$ ) converges in $L^{\infty}$ weak- $\star$ to a function $f \in L^{\infty}\left(\Omega_{l} \times V \times[0, T]\right)$ and $f$ satisfies Eq. (34).

(ii) A subsequence of $f_{\epsilon}\left(\right.$ still denoted $\left.f_{\epsilon}\right)$ converges strongly in $L^{1}\left(\Omega_{l} \times V \times(0, T)\right)$ to a function $f \in L^{1}\left(\Omega_{l} \times V \times(0, T)\right)$ and $u=\int_{V} f$.

The proof of the first point in (ii) of Theorem 4.3 relies also on the following result [26].

Theorem 4.4. Let $U$ be a bounded open subset of $\mathbb{R}^{N}$ and let $v_{n}$ be a sequence in $L_{l o c}^{1}(U)$. Assume that as $n \rightarrow \infty$, the sequence $v_{n}$ converges strongly in $L_{l o c}^{1}(U)$ to $v \in L_{l o c}^{1}(U)$. If $v_{n}$ is uniformly bounded in $L^{\infty}(U)$, then $v_{n}$ converges strongly to $v$ in $L^{1}(U)$.

Proof of Theorem 4.3. (i) Using Theorem 4.17a subsequence of $u_{\epsilon}$ converges strongly in $L^{1}\left(\Omega_{l} \times(0, T)\right)$ to a function $u$. Hence by the properties of $\chi, \chi_{u_{\epsilon}}$ converges strongly to $\chi_{u}$ in $L^{1}\left(\Omega_{l} \times V \times(0, T)\right.$ ). Using Lemma 2.1] a subsequence of $f_{\epsilon}$ (still denoted $\left.f_{\epsilon}\right)$ converges, as $\epsilon \rightarrow 0$, in $L^{\infty}\left(\Omega_{l} \times V \times[0, T]\right)$ weak- $\star$ to a function $f \in L^{\infty}\left(\Omega_{l} \times V \times[0, T]\right)$. Moreover, $\left(\partial_{t}+a(v) \cdot \partial_{x}\right) f_{\epsilon}$ converges to $\left(\partial_{t}+a(v) \cdot \partial_{x}\right) f$ in the distribution sense. We also know from above and from the diagonal process to subtract a further subsequence, if necessary, that a subsequence of $\chi_{u_{\epsilon}}$ converges as $\epsilon \rightarrow 0$ to $\chi_{u}$ strongly in $L^{1}\left(\Omega_{l} \times V \times(0, T)\right)$ and that $f \in L^{\infty}\left(\Omega_{l} \times V \times[0, T]\right)$. Therefore $f$ satisfies Eq. (34) a.e. 
(ii) The function $f_{\epsilon}$ satisfies Eq. (11). Subtracting this equation from Eq. (34), and multiplying the resulting equation by $\varphi$, a test function in $C^{1}\left(\Omega_{l} \times V \times[0, T]\right)$, with compact support in $x$ to be made precise later, and integrating by parts, we obtain

$$
\begin{aligned}
& \int_{\Omega_{l} \times V}\left(\left(f_{\epsilon}-f\right) \varphi\right)(\cdot, \cdot, t)-\int_{\Omega_{l} \times V}\left(\left(f_{\epsilon}-f\right) \varphi\right)(\cdot, \cdot, 0) \\
& -\int_{\Omega_{l} \times V \times(0, t)}\left(\partial_{t}+a(v) \cdot \partial_{x}\right)(\varphi)\left(f_{\epsilon}-f\right) \\
= & \frac{1}{\epsilon_{l}} \int_{\Omega_{l} \times V \times(0, t)}\left(\left(\chi_{u_{\epsilon}}-\chi_{u}\right)-\left(f_{\epsilon}-f\right)\right) \varphi .
\end{aligned}
$$

We recall that the trace of $f$ at the initial time exists since $\left(\partial_{t}+a(v) \cdot \partial_{x}\right) f$ and $f$ are in $L^{1} \cap L^{\infty}$. We then take $\varphi=\operatorname{sign}^{\mu}\left(f_{\epsilon}-f\right) \psi(x, t)$ with $x \operatorname{sign}^{\mu}(x) \geq 0$, $x \in \mathbb{R}$, where $\psi$ is a nonnegative test function and $\operatorname{sign}^{\mu}$ is a regularization of sign function. Plugging in (49) and passing to the limit as $\mu \rightarrow 0$, we obtain

$$
\begin{aligned}
& \int_{\Omega_{l} \times V}\left(\left|f_{\epsilon}-f\right| \psi\right)(\cdot, \cdot, t)+\frac{1}{\epsilon_{l}} \int_{\Omega_{l} \times V \times(0, t)}\left|f_{\epsilon}-f\right| \psi \\
= & \frac{1}{\epsilon_{l}} \int_{\Omega_{l} \times V \times(0, t)}\left(\chi_{u_{\epsilon}}-\chi_{u}\right) \operatorname{sign}\left(f_{\epsilon}-f\right) \psi+\int_{\Omega_{l} \times V}\left(\left|f_{\epsilon}-f\right| \psi\right)(\cdot, \cdot, 0) \\
& +\int_{\Omega_{l} \times V \times(0, t)}\left(\partial_{t}+a(v) \cdot \partial_{x}\right)(\psi)\left|f_{\epsilon}-f\right| .
\end{aligned}
$$

In particular we have

$$
\begin{aligned}
& \int_{O \times V}\left(\left|f_{\epsilon}-f\right| \psi\right)(\cdot, \cdot, t)+\frac{1}{\epsilon_{l}} \int_{O \times V \times(0, t)}\left|f_{\epsilon}-f\right| \psi \\
= & \frac{1}{\epsilon_{l}} \int_{O \times V \times(0, t)}\left(\chi_{u_{\epsilon}}-\chi_{u}\right) \operatorname{sign}\left(f_{\epsilon}-f\right) \psi \\
& +\int_{O \times V \times(0, t)}\left(\partial_{t}+a(v) \cdot \partial_{x}\right)(\psi)\left|f_{\epsilon}-f\right|
\end{aligned}
$$

for any open set with $\bar{O} \subset \Omega_{l}$, and $\psi$ for any Lipschitz continuous function in $O \times V \times[0, T]$ with compact support in $x$ in $O$ and such that $\psi(., ., 0) \equiv 0$.

Let $U$ and $O$ be open bounded subsets of $\Omega_{l}$ such that $\bar{U} \subset O \subset \bar{O} \subset \Omega_{l}$. Let $\psi$ be a Lipschitz continuous function in $O \times V \times[0, T]$ with compact support in $x$ in $O$ and satisfying $\psi(., ., 0) \equiv 0$, such that $U \subset \operatorname{supp}_{x} \psi \subset O$. Then (52) holds for such $\psi$ and $O$.

We wish to prove that

$$
\int_{U \times V}\left|f_{\epsilon}-f\right|(\cdot, \cdot, t)+\frac{1}{\epsilon_{l}} \int_{U \times V \times(\eta, t)}\left|f_{\epsilon}-f\right| \rightarrow_{\epsilon \rightarrow 0} 0
$$

for any $t \in(0, T]$ and any $0<\eta<t \leq T$. It is enough to prove this relation for $U$ of the form $U=(y-\alpha, y+\alpha)$, where $\alpha>0$ is any positive real such that $0<y-\alpha<$ $y+\alpha<l$. Let $\beta>0$ and $\gamma>0$ be such that $0<y-\alpha-\beta-\gamma<y+\alpha+\beta+\gamma<l$. Let $0<t_{1}<T$ be such that $a_{\infty} t_{1}=\beta$. Let $O=\left(y-\alpha-a_{\infty} t_{1}-\gamma, y+\alpha+a_{\infty} t_{1}+\gamma\right)$ 
and let $t \in\left(0, t_{1}\right]$. Now consider the function

$$
\psi_{1}(x, \tau)= \begin{cases}0 & 0 \leq x<y-\alpha-a_{\infty}(t-\tau)-\gamma, \\ \frac{1}{\gamma}\left(x-y+\alpha+a_{\infty}(t-\tau)\right)+1 & 0 \leq \tau \leq t, \\ & y-\alpha-a_{\infty}(t-\tau)-\gamma \leq x \\ & <y-\alpha-a_{\infty}(t-\tau), 0 \leq \tau \leq t, \\ & y-\alpha-a_{\infty}(t-\tau) \leq x<y+\alpha \\ & +a_{\infty}(t-\tau), 0 \leq \tau \leq t, \\ \frac{1}{\gamma}\left(y+\alpha+a_{\infty}(t-\tau)-x\right)+1 & y+\alpha+a_{\infty}(t-\tau) \leq x<y+\alpha \\ & +a_{\infty}(t-\tau)+\gamma, 0 \leq \tau \leq t, \\ 0 & y+\alpha+a_{\infty}(t-\tau)+\gamma \leq x \leq l, \\ & 0 \leq \tau \leq t,\end{cases}
$$

and

$$
\psi_{2}(x, \tau)=e^{-\frac{1}{\tau}}
$$

Now let $\psi(x, \tau)=\psi_{1}(x, \tau) \psi_{2}(x, \tau), \tau \in[0, t]$, and $x \in \Omega_{l}$. It is clear that $\psi$ is a nonnegative Lipschitz continuous function in $O \times V \times[0, T]$ with compact support in $x$ in $O$ and $U \subset \operatorname{supp}_{x} \psi \subset O$. Thus, plugging $\psi$ in (52) and using the fact that $\chi_{u_{\epsilon}}$ converges strongly in $L^{1}\left(\Omega_{l} \times V \times(0, T)\right)$ to $\chi_{u}$ yields (52) for $t \in\left(0, t_{1}\right]$. Now let $t_{2}>t_{1}$ be such that $a_{\infty}\left(t_{2}-t_{1}\right)=\beta$. Proceeding as above and using the fact that $\int_{U \times V}\left|f_{\epsilon}-f\right|\left(\cdot, \cdot, t_{1}\right)$ converges to 0 as $\epsilon \rightarrow 0$, we conclude that $\int_{U \times V}\left|f_{\epsilon}-f\right|(\cdot, \cdot, t)$ and $\frac{1}{\epsilon_{l}} \int_{U \times V \times\left(t_{1}, t_{2}\right)}\left|f_{\epsilon}-f\right|$ converge to 0 for any $t \in\left(t_{1}, t_{2}\right]$. Continuing this process we conclude that $\int_{U \times V}\left|f_{\epsilon}-f\right|(\cdot, \cdot, t)$ and $\frac{1}{\epsilon_{l}} \int_{U \times V \times(\eta, T)}\left|f_{\epsilon}-f\right|$ converge to 0 for any $t \in(0, T]$ and any $0<\eta<t \leq T$.

Therefore, $f_{\epsilon}$ converges in $L_{l o c}^{1}\left(\Omega_{l} \times(0, T) ; L^{1}(V)\right) \cap L^{\infty}\left([\eta, T] ; L_{l o c}^{1}\left(\Omega_{l} ; L^{1}(V)\right)\right)$ to $f$. Now using Lemma $2.3 \operatorname{supp}_{v} f_{\epsilon}$ is included into a fixed compact set independent of $\epsilon$. Therefore, since $f_{\epsilon}$ converges to $f$ in $L_{l o c}^{1}$ and $f_{\epsilon}$ is uniformly bounded in $L^{\infty}\left(\Omega_{l} \times V \times[0, T]\right)$ (Lemma 2.1), we may use Theorem 4.4 to infer that $f_{\epsilon}$ converges strongly to $f$ in $L^{1}\left(\Omega_{l} \times V \times(0, T)\right)$.

Finally, since $\int_{\Omega_{l} \times(0, T)}\left|u_{\epsilon}-\int_{V} f\right| \leq \int_{\Omega_{l} \times V \times(0, T)}\left|f_{\epsilon}-f\right|$ and since the limit is unique, $u=\int_{V} f$. The proof of the theorem is now completed.

Remark 4.1. Notice that in the course of proving Theorem 4.3, we have proved that $f_{\epsilon}$ converges strongly to $f$ in $L^{\infty}\left([\eta, T] ; L^{1}\left(\Omega_{l} \times V\right)\right)$ for any $0<\eta<T$.

Theorem 4.5. Assume that

$$
\begin{aligned}
& \left\|f_{\epsilon 0}\right\|_{L^{\infty}\left(\Gamma_{1}^{-} \times[0, T]\right)}<C_{1}, \quad\left\|g_{\epsilon}^{0}\right\|_{L^{\infty}\left(\Omega_{g} \times V\right)}<C_{2}, \quad\left\|f_{\epsilon}^{0}\right\|_{L^{\infty}\left(\Omega_{l} \times V\right)}<C_{3}, \\
& \left\|g_{\epsilon 1}\right\|_{L^{\infty}\left(\Gamma_{2}^{-} \times[0, T]\right)}<C_{4},\left\|f_{\epsilon}^{0}\right\|_{L^{1}\left(\Omega_{l} \times V\right)}<C_{5},\left\|g_{\epsilon}^{0}\right\|_{L^{1}\left(\Omega_{g} \times V\right)}<C_{6}, \\
& \left\|a(v) \cdot n_{l} f_{\epsilon 0}\right\|_{L^{1}\left(\Gamma_{1}^{-} \times(0, T)\right)}<C_{7},\left\|a(v) \cdot n_{g} g_{\epsilon 1}\right\|_{L^{1}\left(\Gamma_{2}^{-} \times(0, T)\right)}<C_{8}
\end{aligned}
$$

with $C_{i}, i=1, \cdots, 8$, positive constants independent of $\epsilon$.

Assume also that the initial and boundary data $f_{\epsilon}^{0}, f_{\epsilon 0}, g_{\epsilon}^{0}$, and $g_{\epsilon 1}$ are compactly supported in $v \in V$ with supports included in a fixed compact set independent of $\epsilon$. Finally assume that (48) holds. Then:

(i) A subsequence of $w_{\epsilon}=\int_{\mathbb{R}} g_{\epsilon} d v$ (still denoted $\left.w_{\epsilon}\right)$ converges in $L_{l o c}^{1}\left(\Omega_{g} \times(0, T)\right.$ ) to a function $w$.

(ii) The strong convergence in $L_{l o c}^{1}$ is actually a strong convergence in $L^{1}$.

(iii) A subsequence of $g_{\epsilon}$ (still denoted $\left.g_{\epsilon}\right)$ converges in $L^{\infty}\left(\Omega_{g} \times V \times(0, T)\right)$ weak-太 to a function $g$ and $g=\chi_{w}$. 
The proof of point (i) in Theorem 4.5 relies on an extension to domains with boundaries of a compactness result introduced in [12]. This general compactness result is a variant of compactness results shown in [7]. The extension result is given in the following theorem.

Theorem 4.6. Let $1<p \leq 2$, let $g$ be bounded in $L_{l o c}^{p}\left(\Omega_{g} \times \mathbb{R}_{v} \times(0, T)\right)$, let $h$ belong to a compact set of $L^{p}\left(\Omega_{g} \times \mathbb{R}_{v} \times(0, T)\right)$, and let $r \geq 0$. We assume that $g$ satisfies

$$
\frac{\partial g}{\partial t}+\sum_{i=1}^{d} a_{i}(v) \frac{\partial g}{\partial x_{i}}=\left(-\Delta_{x, t}+1\right)^{1 / 2}\left(-\Delta_{v}+1\right)^{r / 2} h \quad \text { in } \mathcal{D}^{\prime}
$$

where $a(\cdot) \in C_{\text {loc }}^{s, \alpha}$ with $s=r, \alpha=1$ if $r$ is an integer, $s=[r]$, and $\alpha=r-s$ if $r$ is not an integer. Let $\psi \in L^{p^{\prime}}\left(\mathbb{R}_{v}\right)$ with compact support. Finally, we assume that $a(\cdot)$ satisfies (48). Then, $\int_{\mathbb{R}} g \psi d v$ belongs to a compact set of $L_{l o c}^{p}\left(\Omega_{g} \times(0, T)\right)$.

The proof of this theorem relies on a slight modification of the proof of the related result for the domain $\mathbb{R}^{d} \times \mathbb{R}_{v} \times(0, \infty)$. The proof of the result on the domain $\mathbb{R}^{d} \times \mathbb{R}_{v} \times(0, \infty)$ is given in $[12$ and proved there; see also [7].

The proof of point (ii) in Theorem 4.5 relies on Theorem 4.4

Proof of Theorem 4.5. (i) For the proof of point (i) of this theorem we follow [12]; see also [27] and refer to it for more details. We first notice that using Lemmas 2.1 2.2, $g_{\epsilon}$ is uniformly bounded in $L^{q}\left(\Omega_{g} \times V \times(0, T)\right)$ for any $q \geq 1$. Second, we prove that we can write $\frac{1}{\epsilon}\left(\chi_{w_{\epsilon}(x, t)}(v)-g_{\epsilon}(x, v, t)\right)$ as $\partial m_{\epsilon} / \partial v$ with $m_{\epsilon}$ a nonnegative measure on $\Omega_{g} \times \mathbb{R}_{v} \times(0, T)$ bounded independently of $\epsilon>0$. By applying Sobolev embeddings with $p<(d+2) /(d+1)$, we conclude that $m_{\epsilon}$ is bounded in

$$
W^{-s, p}\left(\Omega_{g} \times \mathbb{R}_{v} \times(0, T)\right)
$$

for $(d+2) / p^{\prime}<s<1$. Then $\partial m_{\epsilon} / \partial v$ can be written in the form of the right-hand side in (53) for $r>1+(d+2) / p^{\prime}$. Taking $\psi(v)=\mathbb{1}_{\left[-R_{\infty}, R_{\infty}\right]}(v)$, we conclude the proof of the first assertion in the theorem.

(ii) We now proceed in proving the second assertion. Using Lemma 2.3 and the diagonal process to extract a further subsequence, if necessary, a subsequence of $w_{\epsilon}$ converges in $L^{\infty}$ weak- $\star$ to a function $w \in L^{\infty}\left(\Omega_{g} \times[0, T]\right)$. Therefore, since $\Omega_{g} \times(0, T)$ is bounded, we can use Theorem 4.4 and conclude that the strong $L_{l o c}^{1}$ convergence of $w_{\epsilon}$ is actually a strong $L^{1}$ convergence.

(iii) Using Lemma 2.1 a subsequence of $g_{\epsilon}$ converges in $L^{\infty}$ weak- $\star$ to a function $g \in L^{\infty}\left(\Omega_{g} \times V \times[0, T]\right)$. The fact that $g=\chi_{w}$ results from using equation (4), the strong $L^{1}$ convergence of $\chi_{w_{\epsilon}}$ to $\chi_{w}$ (through (ii) above and the properties of the function $\chi$ ), the $L^{\infty}$ weak- $\star$ convergence of $g_{\epsilon}$, and the uniqueness of the limit. The theorem is now proved.

Next we give some convergence properties of $g_{\epsilon}$ and their relations to those of $w_{\epsilon}$.

Theorem 4.7. Assume that

$$
\begin{aligned}
& \left\|f_{\epsilon 0}\right\|_{L^{\infty}\left(\Gamma_{1}^{-} \times[0, T]\right)}<C_{1}, \quad\left\|g_{\epsilon}^{0}\right\|_{L^{\infty}\left(\Omega_{g} \times V\right)}<C_{2}, \quad\left\|f_{\epsilon}^{0}\right\|_{L^{\infty}\left(\Omega_{l} \times V\right)}<C_{3}, \\
& \left\|g_{\epsilon 1}\right\|_{L^{\infty}\left(\Gamma_{2}^{-} \times[0, T]\right)}<C_{4}, \quad\left\|f_{\epsilon}^{0}\right\|_{L^{1}\left(\Omega_{l} \times V\right)}<C_{5}, \quad\left\|g_{\epsilon}^{0}\right\|_{L^{1}\left(\Omega_{g} \times V\right)}<C_{6}, \\
& \left\|a(v) \cdot n_{l} f_{\epsilon 0}\right\|_{L^{1}\left(\Gamma_{1}^{-} \times(0, T)\right)}<C_{7}, \quad\left\|a(v) \cdot n_{g} g_{\epsilon 1}\right\|_{L^{1}\left(\Gamma_{2}^{-} \times(0, T)\right)}<C_{8}
\end{aligned}
$$


with $C_{i}, i=1, \cdots, 8$, positive constants independent of $\epsilon$.

Assume also that the initial and boundary data $f_{\epsilon}^{0}, f_{\epsilon 0}, g_{\epsilon}^{0}$, and $g_{\epsilon 1}$ are compactly supported in $v \in V$ with supports included in a fixed compact set independent of $\epsilon$. Finally assume that (48) holds. Then

(i) For any real $k$,

$$
\int_{V}\left|g_{\epsilon}-\chi_{k}\right| d v-\left|w_{\epsilon}-k\right| \rightarrow_{\epsilon \rightarrow 0} 0 \text { in } L_{l o c}^{1}\left(\Omega_{g} \times(0, T)\right) .
$$

(ii) For any $b(\cdot) \in L^{\infty}(V)$,

$$
\int_{V} b(v)\left|g_{\epsilon}-\chi_{k}\right| d v-\operatorname{sgn}\left(w_{\epsilon}-k\right) \int_{V} b(v)\left(g_{\epsilon}-\chi_{k}\right) d v \rightarrow_{\epsilon \rightarrow 0} 0 \text { in } L_{l o c}^{1}\left(\Omega_{g} \times(0, T)\right) \text {. }
$$

$$
\overline{\int_{V}\left|g_{\epsilon}-\chi_{k}\right| d v}=\overline{\left|w_{\epsilon}-k\right|}=|w-k| .
$$

(iv)

$$
\begin{aligned}
\overline{\int_{V} a_{i}(v)\left|g_{\epsilon}-\chi_{k}\right| d v} & =\overline{\operatorname{sgn}\left(w_{\epsilon}-k\right) \int_{V} a_{i}(v)\left(g_{\epsilon}-\chi_{k}\right)} \\
& =\operatorname{sgn}(w-k)\left(A_{i}(w)-A_{i}(k)\right) \\
& =\int_{V} a_{i}(v)\left|\chi_{w}-\chi_{k}\right| .
\end{aligned}
$$

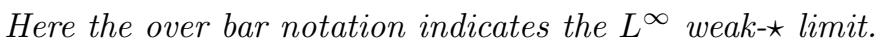

Proof of Theorem 4.7. The proofs of (i) and (ii) are exactly the same as the proof of Lemma 3.4 of [16. The proofs of (iii) and (iv) are similar to the proofs of (3.17), (3.18), and (3.19) of [16]. In [16], the authors make the assumption that a subsequence of $w_{\epsilon}$ (in their notations $u_{\epsilon}$ ) converges strongly to $w: w_{\epsilon} \rightarrow w$ (in their notations $u_{\epsilon} \rightarrow u$; see (3.9) in [16]). Here we know from Theorem 4.5] that $w_{\epsilon}$ converges strongly in $L^{1}\left(\Omega_{g} \times V \times(0, T)\right)$ to $w$. Hence in our case the use of $(3.9)$ in 16. must be replaced by the use of Theorem 4.5. Also in our case the justification of the weak- $\star L^{\infty}$-limit of the various quantities (after extraction of appropriate subsequences, if necessary) results from Lemmas 2.1 and 2.3

Next, we state a result about the existence of a solution to the kinetic formulation of the coupled system given in (34)-(42).

Theorem 4.8. Assume that

$$
\begin{aligned}
& \left\|f_{\epsilon 0}\right\|_{L^{\infty}\left(\Gamma_{1}^{-} \times[0, T]\right)}<C_{1}, \quad\left\|g_{\epsilon}^{0}\right\|_{L^{\infty}\left(\Omega_{g} \times V\right)}<C_{2}, \quad\left\|f_{\epsilon}^{0}\right\|_{L^{\infty}\left(\Omega_{l} \times V\right)}<C_{3}, \\
& \left\|g_{\epsilon 1}\right\|_{L^{\infty}\left(\Gamma_{2}^{-} \times[0, T]\right)}<C_{4}, \quad\left\|f_{\epsilon}^{0}\right\|_{L^{1}\left(\Omega_{l} \times V\right)}<C_{5},\left\|g_{\epsilon}^{0}\right\|_{L^{1}\left(\Omega_{g} \times V\right)}<C_{6}, \\
& \left\|a(v) \cdot n_{l} f_{\epsilon 0}\right\|_{L^{1}\left(\Gamma_{1}^{-} \times(0, T)\right)}<C_{7}, \quad\left\|a(v) \cdot n_{g} g_{\epsilon 1}\right\|_{L^{1}\left(\Gamma_{2}^{-} \times(0, T)\right)}<C_{8}
\end{aligned}
$$

with $C_{i}, i=1, \cdots, 8$, positive constants independent of $\epsilon$.

Assume also that the initial and boundary data $f_{\epsilon}^{0}, f_{\epsilon 0}, g_{\epsilon}^{0}$, and $g_{\epsilon 1}$ are compactly supported in $v \in V$ with supports included in a fixed compact set independent of $\epsilon$. 
Finally assume that (48) hold and that as $\epsilon \rightarrow 0$,

$$
\begin{aligned}
& \left\|g_{\epsilon}^{0}(\cdot, \cdot)-\chi_{w^{0}(\cdot)}(\cdot)\right\|_{L_{l o c}^{1}\left(\Omega_{g} \times L^{1}(V)\right)} \rightarrow 0, \\
& f_{\epsilon}^{0} \rightarrow f^{0} \text { strongly in } L^{1}\left(\Omega_{l} \times V\right), \\
& a(v) \cdot n f_{\epsilon 0} \rightarrow a(v) \cdot n f_{0} \text { weakly in } L^{1}\left(\Gamma_{1}^{-} \times(0, T)\right), \\
& a(v) \cdot n g_{\epsilon 1} \rightarrow a(v) \cdot n g_{1}=a(v) \cdot n \chi_{w_{1}} \text { strongly in } L^{1}\left(\Gamma_{2}^{-} \times(0, T)\right) .
\end{aligned}
$$

Then $f_{\epsilon}$ converges strongly in $L^{1}\left(\Omega_{l} \times V \times(0, T)\right)$, as $\epsilon$ goes to 0 , to $f$, and $g_{\epsilon}$ converges in $L^{\infty}\left(\Omega_{g} \times V \times(0, T)\right)$ weak- $\star$ to $g$, and $(f, g)$ is a solution of the system (34) - (42) in the sense of Definition 3.2 .

Remark 4.2. A fundamental point in Definitions 3.1 and 3.2 of solutions to the coupled systems is the precise statement of the interface conditions between the kinetic model and its hydrodynamic conservation laws limit. The rigorous derivation and justification of such interface conditions is given in the following theorems.

Theorem 4.9. For any $\psi_{1} \in C_{0}^{1}\left(\bar{\Omega}_{l} \times V \times(0, T)\right)$ and for any $k \in \mathbb{R}$, we have

$$
\lim _{\epsilon \rightarrow 0} \int_{\Gamma_{i}^{+} \times(0, T)} a(v) \cdot n \psi_{1}\left|f_{\epsilon}-\chi_{k}\right|=\int_{\Gamma_{i}^{+} \times(0, T)} a(v) \cdot n \psi_{1}\left|f-\chi_{k}\right| .
$$

Theorem 4.10. For any $\psi_{2} \in C_{0}^{1}\left(\bar{\Omega}_{g} \times V \times(0, T)\right)$, we have

$$
\lim _{\epsilon \rightarrow 0} \int_{\Gamma_{i}^{-} \times(0, T)} a(v) \cdot n \psi_{2} g_{\epsilon}=\int_{\Gamma_{i}^{-} \times(0, T)} a(v) \cdot n \psi_{2} \chi_{w} .
$$

The proofs of Theorems 4.914.10 will be given at the end of this section. The proof of Theorem 4.10 relies on a new regularity theory introduced in this paper; see also the author's papers [27, [25].

Remark 4.3. Theorem 4.8 is obtained under various assumptions including the assumption that the data $f_{\epsilon}^{0}, f_{\epsilon 0}, g_{\epsilon}^{0}$, and $g_{\epsilon 1}$ are compactly supported in $v$. In fact this theorem is also valid when these data are not necessarily compactly supported in $v$. The proof is based on a BV-regularization argument.

Proof of Theorem 4.8. Using Theorems 4.1, 4.3, and 4.5 a subsequence of $f_{\epsilon}, u_{\epsilon}$, and $w_{\epsilon}$ (still denoted $f_{\epsilon}, u_{\epsilon}$, and $w_{\epsilon}$ ) converges strongly in $L^{1}$ resp. to $f, u$, and $w$. Now using Theorem 4.5 a subsequence of $g_{\epsilon}$ (still denoted $g_{\epsilon}$ ) converges in $L^{\infty}$ weak- $\star$ to $g=\chi_{w}$. It is clear that

$$
\begin{aligned}
(f, g) \in & \left(L^{\infty}\left([0, T] ; L^{1}\left(\Omega_{l} \times V\right)\right) \cap L^{\infty}\left(\Omega_{l} \times[0, T] ; L^{1}(V)\right)\right) \\
& \times\left(L^{\infty}\left([0, T] ; L^{1}\left(\Omega_{g} \times V\right)\right) \cap L^{\infty}\left(\Omega_{g} \times(0, T) ; L^{1}(V)\right)\right) .
\end{aligned}
$$

We shall first prove that the first assertion in Definition 3.2 is satisfied; that is, we wish to prove that Eq. (37) is satisfied. Using the above we know that $\left[\partial_{t}+a(v) \cdot \partial_{x}\right] g_{\epsilon}(x, v, t)$ converges to $\left[\partial_{t}+a(v) \cdot \partial_{x}\right] g(x, v, t)$ in the distribution sense. Proceeding as in the proof of Theorem 4.5] and following [12] (see also [27]) we can write $\frac{1}{\epsilon}\left(\chi_{w_{\epsilon}(x, t)}(v)-g_{\epsilon}(x, v, t)\right)$ as $\partial m_{\epsilon} / \partial v$ with $m_{\epsilon}$ a nonnegative measure on $\Omega_{g} \times \mathbb{R}_{v} \times(0, T)$ bounded independently of $\epsilon>0$ and which converges to the unique measure $m$ given in (37); i.e.

$$
\left[\partial_{t}+a(v) \cdot \partial_{x}\right] g(x, v, t)=\frac{\partial m}{\partial v} \text { in } \Omega_{g} \times V \times(0, T) .
$$


Hence the equation (37) is satisfied. We now prove the second part in Definition 3.2. Using Theorem [2.2, we have

$$
\begin{aligned}
& -\int_{\Omega_{g} \times V \times(0, T)}\left(\partial_{t}+a(v) \cdot \partial_{x}\right)\left(\psi_{2}\right)\left|g_{\epsilon}-\chi_{k}\right|+\int_{\Gamma_{i}^{+} \times(0, T)} a(v) \cdot n_{g} \psi_{2}\left|f_{\epsilon}-\chi_{k}\right| \\
& +\int_{\Gamma_{2}^{-} \times(0, T)} a(v) \cdot n_{g} \psi_{2}\left|g_{\epsilon 1}-\chi_{k}\right| \leq 0 \quad \forall \psi_{2} \in C_{0}^{1}\left(\bar{\Omega}_{g} \times(0, T)\right), \psi_{2} \geq 0, \forall k \in \mathbb{R} .
\end{aligned}
$$

Using Theorems 4.5, 4.7, and 4.9, and (57), and letting $\epsilon \rightarrow 0$ in the above inequality, we obtain

$$
\begin{aligned}
& -\int_{\Omega_{g} \times V \times(0, T)}\left(\partial_{t}+a(v) \cdot \partial_{x}\right)\left(\psi_{2}\right)\left|g-\chi_{k}\right|+\int_{\Gamma_{i}^{+} \times(0, T)} a(v) \cdot n_{g} \psi_{2}\left|f-\chi_{k}\right| \\
& +\int_{\Gamma_{2}^{-} \times(0, T)} a(v) \cdot n_{g} \psi_{2}\left|\chi_{w_{1}}-\chi_{k}\right| \leq 0 \quad \forall \psi_{2} \in C_{0}^{1}\left(\bar{\Omega}_{g} \times(0, T)\right), \psi_{2} \geq 0, \forall k \in \mathbb{R}
\end{aligned}
$$

with $g=\chi_{w}$.

For the local problem (problem in $\Omega_{l}$ ) we know that $f$ satisfies (34); see Theorem 4.3. On the other hand $f_{\epsilon}$ satisfies for any $\psi_{1} \in C_{0}^{1}\left(\bar{\Omega}_{l} \times V \times(0, T)\right)$

$$
\begin{aligned}
& -\int_{\Omega_{l} \times V \times(0, T)}\left(\partial_{t}+a(v) \cdot \partial_{x}\right)\left(\psi_{1}\right) f_{\epsilon}+\int_{\Gamma_{1}^{-} \times(0, T)} a(v) \cdot n \psi_{1} f_{\epsilon 0} \\
& +\int_{\Gamma_{1}^{+} \times(0, T)} a(v) \cdot n \psi_{1} f_{\epsilon}+\int_{\Gamma_{i}^{-} \times(0, T)} a(v) \cdot n \psi_{2} g_{\epsilon} \\
& +\int_{\Gamma_{i}^{+} \times(0, T)} a(v) \cdot n \psi_{2} f_{\epsilon}=\frac{1}{\epsilon_{l}} \int_{\Omega_{l} \times V \times(0, T)}\left(\chi_{u_{\epsilon}}-f_{\epsilon}\right) \psi_{1} .
\end{aligned}
$$

Using Theorems 4.1 and 4.3, together with the properties of $\chi$, the first term converges to

$$
-\int_{\Omega_{l} \times V \times(0, T)}\left(\partial_{t}+a(v) \cdot \partial_{x}\right)\left(\psi_{1}\right) f
$$

and the right side in (61) converges to

$$
\frac{1}{\epsilon_{l}} \int_{\Omega_{l} \times V \times(0, T)}\left(\chi_{u}-f\right) \psi_{1} .
$$

Using (56), the second term in (61) converges to

$$
\int_{\Gamma_{1}^{-} \times(0, T)} a(v) \cdot n \psi_{1} f_{0} .
$$

Now let $\psi_{1} \in C_{0}^{1}\left(\bar{\Omega}_{l} \times V \times(0, T)\right)$ be such that $\psi_{1} \equiv 0$ on $\Gamma_{1} \times V \times(0, T)$ and on $\Gamma_{i}^{-} \times(0, T)$. Multiplying equation (34) by $\psi_{1}$ and integrating by parts, we obtain

$$
\begin{aligned}
\int_{\Gamma_{i}^{+} \times(0, T)} a(v) \cdot n \psi_{1} f= & \int_{\Omega_{l} \times V \times(0, T)}\left(\partial_{t}+a(v) \cdot \partial_{x}\right)\left(\psi_{1}\right) f \\
& +\frac{1}{\epsilon_{l}} \int_{\Omega_{l} \times V \times(0, T)}\left(\chi_{u}-f\right) \psi_{1} .
\end{aligned}
$$


On the other hand multiplying Eq. (1) by $\psi_{1}$ and integrating by parts, we obtain

$$
\begin{aligned}
\int_{\Gamma_{i}^{+} \times(0, T)} a(v) \cdot n \psi_{1} f_{\epsilon}= & \int_{\Omega_{l} \times V \times(0, T)}\left(\partial_{t}+a(v) \cdot \partial_{x}\right)\left(\psi_{1}\right) f_{\epsilon} \\
& +\frac{1}{\epsilon_{l}} \int_{\Omega_{l} \times V \times(0, T)}\left(\chi_{u_{\epsilon}}-f_{\epsilon}\right) \psi_{1} .
\end{aligned}
$$

Thanks to (62) and (63), letting $\epsilon \rightarrow 0$ in (66) and using (65), we obtain

$$
\operatorname{limit}_{\epsilon \rightarrow 0} \int_{\Gamma_{i}^{+} \times(0, T)} a(v) \cdot n \psi_{1} f_{\epsilon}=\int_{\Gamma_{i}^{+} \times(0, T)} a(v) \cdot n \psi_{1} f .
$$

By a similar method, we obtain

$$
\operatorname{limit}_{\epsilon \rightarrow 0} \int_{\Gamma_{1}^{+} \times(0, T)} a(v) \cdot n \psi_{1} f_{\epsilon}=\int_{\Gamma_{1}^{+} \times(0, T)} a(v) \cdot n \psi_{1} f .
$$

Using the transmission condition (3) and Theorem 4.10, we obtain

$$
\operatorname{limit}_{\epsilon \rightarrow 0} \int_{\Gamma_{i}^{-} \times(0, T)} a(v) \cdot n \psi_{1} f_{\epsilon}=\int_{\Gamma_{i}^{-} \times(0, T)} a(v) \cdot n \psi_{1} \chi_{w} .
$$

Thus, letting $\epsilon \rightarrow 0$ in (61) and using (62), (64), (63), (67), (68), and (69), we obtain

$$
\begin{aligned}
& -\int_{\Omega_{l} \times V \times(0, T)}\left(\partial_{t}+a(v) \cdot \partial_{x}\right)\left(\psi_{1}\right) f+\int_{\Gamma_{1}^{-} \times(0, T)} a(v) \cdot n \psi_{1} f_{0} \\
& +\int_{\Gamma_{1}^{+} \times(0, T)} a(v) \cdot n \psi_{1} f+\int_{\Gamma_{i}^{-} \times(0, T)} a(v) \cdot n \psi_{1} \chi_{w} \\
& +\int_{\Gamma_{i}^{+} \times(0, T)} a(v) \cdot n \psi_{1} f=\frac{1}{\epsilon_{l}} \int_{\Omega_{l} \times V \times(0, T)}\left(\chi_{u}-f\right) \psi_{1} .
\end{aligned}
$$

Finally, thanks to the $L^{1}$ strong convergence of $f_{\epsilon}$ and assumption (155), $f$ satisfies the initial condition in (42). Now set $w_{\epsilon}^{0}=\int_{V} g_{\epsilon}^{0}$. Since $g_{\epsilon}^{0}$ satisfies condition (54) and the support in $x$ of $g_{\epsilon}^{0}$ is included in a fixed compact set independent of $\epsilon$ and $g_{\epsilon}^{0}$ is uniformly bounded in $L^{\infty}\left(\Omega_{g} \times V\right)$, we may use Theorem 4.4 to infer that $g_{\epsilon}^{0}$ converges strongly to $\chi_{w^{0}}$ in $L^{1}\left(\Omega_{g} \times V\right)$. Thus, by the properties of $\chi, w_{\epsilon}^{0}$ converges strongly to $w^{0}$ in $L^{1}$. Moreover, we know from Theorem 4.5 that $w_{\epsilon}$ strongly converges in $L^{1}$ to $w$. We then conclude that $w(\cdot, 0)=w^{0}(\cdot)$ and by the properties of $\chi$ and Theorem 4.5 (iii), $g(\cdot, \cdot, 0)=\chi_{w^{0}(\cdot)}(\cdot)$. Hence, $g$ satisfies the initial condition in (42). Therefore $(f, w)$ is an entropic solution in the sense of Definition 3.2 to the system (34)-(42). The proof of the theorem is now complete.

Proof of Theorem 4.9. Let $\psi_{1} \in C_{0}^{1}\left(\bar{\Omega}_{l} \times V \times(0, T)\right)$ be such that $\psi_{1} \equiv 0$ on $\Gamma_{i}^{-} \times$ $(0, T)$ and $\psi_{1} \equiv 0$ on $\Gamma_{1} \times V \times(0, T)$. Using Theorem 2.2 we have

$$
\begin{aligned}
& \int_{\Gamma_{i}^{+} \times(0, T)} a(v) \cdot n \psi_{1}\left|f_{\epsilon}-\chi_{k}\right| \\
= & \int_{\Omega_{l} \times V \times(0, T)}\left(\partial_{t}+a(v) \cdot \partial_{x}\right)\left(\psi_{1}\right)\left|f_{\epsilon}-\chi_{k}\right| \\
& +\frac{1}{\epsilon_{l}} \int_{\Omega_{l} \times V \times(0, T)}\left(\chi_{u_{\epsilon}}-f_{\epsilon}\right) \psi_{1} \operatorname{sign}\left(f_{\epsilon}-\chi_{k}\right) \quad \forall k \in \mathbb{R} .
\end{aligned}
$$


Using Theorems 4.1 and 4.3 together with the properties of $\chi$, the right side in (71) converges as $\epsilon \rightarrow 0$ to

$$
\begin{aligned}
& \int_{\Omega_{l} \times V \times(0, T)}\left(\partial_{t}+a(v) \cdot \partial_{x}\right)\left(\psi_{1}\right)\left|f-\chi_{k}\right| \\
& +\frac{1}{\epsilon_{l}} \int_{\Omega_{l} \times V \times(0, T)}\left(\chi_{u}-f\right) \psi_{1} \operatorname{sign}\left(f-\chi_{k}\right) \quad \forall k \in \mathbb{R} .
\end{aligned}
$$

Since $f$ satisfies Eq. (34) (Theorem 4.3) we can define the trace of $f$ and apply Green's formula to equation (34) (Theorems 2.3] and 2.4). Proceeding as in the proof of Theorem 2.2] (see also [25]) we also have

$$
\begin{aligned}
& \int_{\Gamma_{i}^{+} \times(0, T)} a(v) \cdot n \psi_{1}\left|f-\chi_{k}\right| \\
= & \int_{\Omega_{l} \times V \times(0, T)}\left(\partial_{t}+a(v) \cdot \partial_{x}\right)\left(\psi_{1}\right)\left|f-\chi_{k}\right| \\
& +\frac{1}{\epsilon_{l}} \int_{\Omega_{l} \times V \times(0, T)}\left(\chi_{u}-f\right) \psi_{1} \operatorname{sign}\left(f-\chi_{k}\right) \quad \forall k \in \mathbb{R} .
\end{aligned}
$$

Therefore taking the limit as $\epsilon \rightarrow 0$ in (771) and using (722) and (733) we obtain

$$
\lim _{\epsilon \rightarrow 0} \int_{\Gamma_{i}^{+} \times(0, T)} a(v) \cdot n \psi_{1}\left|f_{\epsilon}-\chi_{k}\right|=\int_{\Gamma_{i}^{+} \times(0, T)} a(v) \cdot n \psi_{1}\left|f-\chi_{k}\right|
$$

which corresponds to (58).

Proof of Theorem 4.10. The proof is split into 5 steps.

Step 1. We give the equation satisfied by the function $g$.

We already know that $g_{\epsilon}$ is uniformly bounded in $L^{1}$ and $L^{\infty}$ (Lemmas 2.1] 2.2, and 2.3) and the limit $g=\chi_{w}$ (see Theorem 4.5(iii)) is in $L^{1}$. Moreover, $\left[\partial_{t}+a(v) \cdot \partial_{x}\right] g_{\epsilon}(x, v, t)$ converges to $\left[\partial_{t}+a(v) \cdot \partial_{x}\right] g(x, v, t)$ in the distribution sense. Proceeding as in [12] and [27], we can write $\frac{1}{\epsilon}\left(\chi_{w_{\epsilon}(x, t)}(v)-g_{\epsilon}(x, v, t)\right)$ as $\partial m_{\epsilon} / \partial v$ and equation (4) in the form

$$
\left[\partial_{t}+a(v) \cdot \partial_{x}\right] g_{\epsilon}(x, v, t)=\frac{\partial m_{\epsilon}}{\partial v} \text { in } \Omega_{g} \times V \times(0, T)
$$

with $m_{\epsilon}$ a nonnegative measure on $\Omega_{g} \times V \times(0, T)$ bounded independently of $\epsilon>0$ and which converges to the unique measure $m$ given by

$$
\left[\partial_{t}+a(v) \cdot \partial_{x}\right] g(x, v, t)=\frac{\partial m}{\partial v} \text { in } \Omega_{g} \times V \times(0, T)
$$

and whose support in the variable $v \in V$ is included by Lemma 2.3 in a compact set of $V\left[-R_{0}, R_{0}\right]$ (since the supports of the measures $m_{\epsilon}$ are included by the same lemma in a fixed compact set independent of $\epsilon$ ). In fact, we can deduce from the above equation and the form of $g$ that the support in $v \in V$ of $m$ is actually included in $[-\tilde{R}, \tilde{R}]$, where $\tilde{R}=\|w\|_{\infty}$. For more on this consult [12]. We shall take $R=\max \left(R_{0}, \tilde{R}\right)$; then the support of $m_{\epsilon}, g_{\epsilon}, \chi_{w_{\epsilon}}$ and $m$ are all contained in the fixed compact set $[-R, R]$.

Step 2. We prove the existence of the normal trace of the flux $g a(v)$ on the boundary.

Let $\psi \in C_{0}^{1}\left(\bar{\Omega}_{g} \times(0, T)\right)$ with $\psi \equiv 0$ on $\Gamma_{2} \times(0, T)$ and

$$
V^{-}=\left\{v \in V: a(v) \cdot n_{g}(x)>0 \text { for some } x \in \Gamma_{i}\right\} \text {. }
$$


Notice that $\Gamma_{i} \times V^{-}=\Gamma_{i}^{-}$.

Let $\varphi \in C_{0}^{1}(V)$ with $\varphi \equiv 0$ on $V \backslash V^{-}$be fixed. Let $F=\left(\int_{V} g \varphi, \int_{V} a_{1}(v) g \varphi, \cdots\right.$, $\left.\int_{V} a_{d}(v) g \varphi\right)$. It is clear from the proof of Theorem 4.5 that $F \in L^{\infty}\left(\Omega_{g} \times(0, T)\right)$. We shall prove that $\operatorname{div}_{t, x} F=\partial_{t} \int_{V} g \varphi+\sum_{i=1}^{d} \partial_{x_{i}} \int_{V} a_{i}(v) g \varphi$ is a bounded measure.

Let $\psi \in C_{0}^{1}\left(\Omega_{g} \times(0, T)\right)$. Using Eq. (76), we obtain

$$
\left\langle\left[\partial_{t}+a(v) \cdot \partial_{x}\right] g, \varphi \otimes \psi\right\rangle=-\int_{\Omega_{g} \times V \times(0, T)} \partial_{v} \varphi \psi d m .
$$

Thus we have

$$
\left\langle\operatorname{div}_{t, x} F, \psi\right\rangle=-\int \partial_{v} \varphi \psi d m .
$$

Since $m$ is a bounded measure on $\Omega_{g} \times V \times(0, T)$ and $\partial_{v} \varphi \in C_{0}(V), \operatorname{div}_{t, x} F$ is also a bounded measure on $\Omega_{g} \times(0, T)$. Now $F \in L^{\infty}\left(\Omega_{g} \times[0, T]\right)$ and $\operatorname{div}_{t, x} F$ is a bounded measure. Therefore, we can apply the Gauss-Green formula of Theorem 2.5 with $U=\Omega_{g} \times(0, T)$ and we obtain in particular

$$
\begin{aligned}
\left\langle\left. F \cdot \nu\right|_{\partial U}, \psi\right\rangle & =\int_{U} \psi \operatorname{div}_{t, x} F+\int_{U} \nabla_{t, x} \psi \cdot F \quad \forall \psi \in C_{0}^{1}\left(\bar{\Omega}_{g} \times(0, T)\right) \\
& =\operatorname{ess~limit}_{z \rightarrow 0} \int_{\partial U_{z}} \psi \circ \Psi_{z}^{-1} F \cdot \nu d \mathcal{H}^{1} .
\end{aligned}
$$

Here $\nabla_{t, x}=\left(\partial_{t}, \partial_{x}\right)$ and $\nu(u)$ is the unit outward normal to $\partial U_{z}$ at $u \in \partial U_{z}$. This is equivalent to

$$
\begin{aligned}
& -\int_{\Omega_{g} \times V \times(0, T)} \partial_{v} \varphi \psi d m+\int_{\Omega_{g} \times V \times(0, T)} g \varphi\left[\partial_{t}+a(v) \cdot \partial_{x}\right] \psi \\
& =\operatorname{ess} \operatorname{limit}_{z \rightarrow 0} \int_{\partial U_{z}} \psi \circ \Psi_{z}^{-1} F \cdot \nu d \mathcal{H}^{1} \text {. }
\end{aligned}
$$

Taking $\psi \in C_{0}^{1}\left(\bar{\Omega}_{g} \times(0, T)\right)$ with $\psi \equiv 0$ on $\partial U \backslash\left(\Gamma_{i} \times(0, T)\right)$, the above equations yield

$$
\begin{aligned}
& -\int \partial_{v} \varphi \psi d m+\int_{\Omega_{g} \times V \times(0, T)} g \varphi\left[\partial_{t}+a(v) \cdot \partial_{x}\right] \psi \\
& =\left\langle\left. F \cdot \nu\right|_{\Gamma_{i} \times(0, T)}, \psi\right\rangle
\end{aligned}
$$

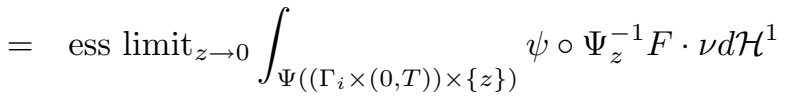

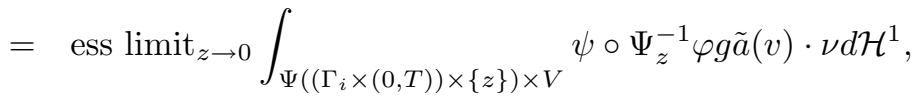

where $\tilde{a}(v)=\left(a_{0}(v), a(v)\right)=(1, a(v))$.

Step 3. We prove the convergence of the traces on $\Gamma_{i}^{-} \times(0, T)$.

Using the integral representation Theorem 2.1 we infer that $g_{\epsilon}$ converges $L^{\infty}$ weakly star on $\Gamma_{i}^{-} \times(0, T)$ to a function $\tilde{g} \in L^{\infty}\left(\Gamma_{i}^{-} \times(0, T)\right)$. Hence, we have

$$
\lim _{\epsilon \rightarrow 0} \int_{\Gamma_{i}^{-} \times(0, T)} a(v) \cdot n \phi g_{\epsilon}=\int_{\Gamma_{i}^{-} \times(0, T)} a(v) \cdot n \phi \tilde{g}, \quad \forall \phi \in L^{1}\left(\Gamma_{i}^{-} \times(0, T)\right),
$$


where we have also used Lemmas 2.3. In particular, we have

$$
\lim _{\epsilon \rightarrow 0} \int_{\Gamma_{i}^{-} \times(0, T)} a(v) \cdot n \varphi \psi g_{\epsilon}=\int_{\Gamma_{i}^{-} \times(0, T)} a(v) \cdot n \varphi \psi \tilde{g}
$$

$\forall \varphi \in C_{0}^{1}(V)$, with $\varphi \equiv 0$ on $V \backslash V^{-}$, and $\forall \psi \in C_{0}^{1}\left(\bar{\Omega}_{g} \times(0, T)\right)$.

Step 4. Let $\psi \in C_{0}^{1}\left(\bar{\Omega}_{g} \times(0, T)\right)$ with $\psi \equiv 0$ on $\left(\partial \Omega_{g} \times(0, T)\right) \backslash\left(\Gamma_{i} \times(0, T)\right)$. It is clear that applying Theorem 2.4 to equation (4) for $g_{\epsilon}$, we obtain

$$
\begin{aligned}
& \int_{\Omega_{g} \times V \times(0, T)} \varphi \psi\left[\partial_{t}+a(v) \cdot \partial_{x}\right] g_{\epsilon}+\int_{\Omega_{g} \times V \times(0, T)} g_{\epsilon} \varphi\left[\partial_{t}+a(v) \cdot \partial_{x}\right] \psi \\
(83)= & -\int_{\Omega_{g} \times V \times(0, T)} \partial_{v} \varphi \psi d m_{\epsilon}+\int_{\Omega_{g} \times V \times(0, T)} g_{\epsilon} \varphi\left[\partial_{t}+a(v) \cdot \partial_{x}\right] \psi \\
(84)= & \int_{\Gamma_{i}^{-} \times(0, T)} a(v) \cdot n \varphi \psi g_{\epsilon} .
\end{aligned}
$$

In this step, we shall prove that the expression in (83) converges to the expression in (79). It is enough to prove such statement for $\psi \in C_{0}^{1}\left(\bar{\Omega}_{g} \times(0, T)\right)$ of the form $\psi(x, t)=\tilde{\psi}(x) \phi(t)$ with $\tilde{\psi}(x) \in C_{0}^{1}\left(\bar{\Omega}_{g}\right)$ and $\phi(t) \in C_{0}^{1}(0, T)$.

We shall split the proof into six steps.

4.1. Let $G$ denote the flux $G(x)=\int_{V \times(0, T)} \varphi(v) \phi(t) g a(v) d v d t$ on $\Omega_{g}$. Eq. (76) yields

$$
\partial_{x} G=-\int_{V \times(0, T)} \partial_{v} \varphi(v) \phi(t) d m+\int_{V \times(0, T)} \varphi(v) \partial_{t} \phi(t) g \text { in } \mathcal{D}^{\prime}\left(\Omega_{g}\right),
$$

where $\varphi(v) \in C_{0}^{1}(V)$ with $\varphi \equiv 0$ on $V \backslash V^{-}$and $\phi(t) \in C_{0}^{1}(0, T)$. Similarly, let $G_{\epsilon}$ denote the flux $G_{\epsilon}(x)=\int_{V \times(0, T)} \varphi(v) \phi(t) g_{\epsilon} a(v) d v d t$. By Lemma 2.2. $G_{\epsilon}$ is uniformly (in $\epsilon$ ) bounded in $L^{1}\left(\Omega_{g}\right)$. Eq. (75) yields

$$
\partial_{x} G_{\epsilon}=-\int_{V \times(0, T)} m_{\epsilon} \partial_{v} \varphi(v) \phi(t)+\int_{V \times(0, T)} \varphi(v) \partial_{t} \phi(t) g_{\epsilon} \text { in } \mathcal{D}^{\prime}\left(\Omega_{g}\right)
$$

Now the first term in the right-hand side of (86) is a bounded measure thanks to the fact that $\phi(t)$ is of compact support in $t \in(0, T), \partial_{v} \varphi(v)$ is of compact support in $v \in V$, and $m_{\epsilon}$ is a bounded measure. Thanks to Lemma 2.2, the second term is a function uniformly (in $\epsilon$ ) bounded in $L^{1}\left(\Omega_{g}\right)$. Thus, the distributional derivative of $G_{\epsilon}$ is a bounded measure and hence $G_{\epsilon}$ is of bounded variation in $\Omega_{g}$. In fact, the right-hand side of (86) is also equal to

$$
\int_{V \times(0, T)} \partial_{v} m_{\epsilon} \varphi(v) \phi(t)+\int_{\times V \times(0, T)} \varphi(v) \partial_{t} \phi(t) g_{\epsilon}
$$

which is a function in $L^{1}\left(\Omega_{g}\right)$ since $\partial_{v} m_{\epsilon}=\frac{1}{\epsilon}\left(\chi_{w_{\epsilon}}-g_{\epsilon}\right)$ and both $\chi_{w_{\epsilon}}$ and $g_{\epsilon}$ are in $L^{1}$ (see Step 1). Then $G_{\epsilon}$ is a function of one variable (recall that we assumed $d=1$ and $\left.\Omega_{g}=(l, 1)\right)$ whose distributional derivative is also a function. Therefore, $G_{\epsilon}$ is an absolutely continuous function [17]. 
4.2. We prove that $\partial_{x} G_{\epsilon}$ is uniformly bounded in $L^{1}\left(\Omega_{g}\right)$.

Using the fact that $\partial_{x} G_{\epsilon}$ is a function and (86) we have

$$
\int_{\Omega_{g}}\left|\partial_{x} G_{\epsilon}\right| \leq \int_{\Omega_{g} \times V \times(0, T)} m_{\epsilon}\left|\partial_{v} \varphi(v) \phi(t)\right|+\int_{\Omega_{g} \times V \times(0, T)}\left|\varphi(v) \partial_{t} \phi(t) g_{\epsilon}\right| .
$$

Using the positiveness and uniform boundedness of the measures $m_{\epsilon}$, Lemma 2.2, and the definition of the functions $\varphi(v)$ and $\phi(t)$, the right-hand side in the inequality above is uniformly (in $\epsilon$ ) bounded and so is the left-hand side.

4.3. Let $P$ denote an extension operator from $W^{1,1}\left(\Omega_{g}\right)$ to $W^{1,1}(\mathbb{R})$. We shall prove that for any $\beta \in L^{1}(\mathbb{R})$ with compact support, $\beta \star \partial_{x} P G_{\epsilon}$ converges to $\beta \star \partial_{x} P G$ strongly in $L^{1}(\mathbb{R})$. Such an extension operator $P$ exists [3]. Here we use the same operators $P$ constructed in [3].

Let $H=P G$ and $H_{\epsilon}=P G_{\epsilon}$ (from Step 4.1 above we know that $G_{\epsilon}$ is in $\left.W^{1,1}\left(\Omega_{g}\right)\right)$. Using Steps 4.1 and 4.2 , the definition of $P$, and its properties, we know that $\partial_{x} H_{\epsilon}$ is uniformly bounded in $L^{1}(\mathbb{R})$ and its support is included in a fixed compact set $\left[-R_{1}, R_{1}\right]$ for some $R_{1}>0$ independent of $\epsilon$. Then the set

$$
\mathcal{B}=\left\{\partial_{x} H_{\epsilon}, \epsilon>0\right\}
$$

is a bounded subset of $L^{1}(\mathbb{R})$. Let $\beta(x) \in L^{1}(\mathbb{R})$ with compact support $K_{\beta} \subset \mathbb{R}$. We shall prove that the set

$$
\mathcal{F}=\left\{\beta \star \partial_{x} H_{\epsilon}, \quad \epsilon>0\right\}
$$

is relatively compact in $L^{1}(\omega)$, for all $\omega$ open bounded set of $\mathbb{R}$. The notation $\star$ refers to the convolution of two functions.

Let $\omega$ be an open bounded set of $\mathbb{R}$ such that $\omega$ contains $\overline{\left[-R_{1}, R_{1}\right]+K_{\beta}}$. This implies that $\omega$ contains the support of the convolution $\beta \star \partial_{x} H_{\epsilon}$. Then we know that (a subsequence of) $\beta \star \partial_{x} H_{\epsilon} \rightarrow_{\epsilon \rightarrow 0} h^{\beta, \omega}$ in $L^{1}(\omega)$ for some function $h^{\beta, \omega} \in$ $L^{1}(\omega)$. Because of the restriction above on the supports and the definition of $\omega$, the convergence takes place actually in $L^{1}(\mathbb{R})$. We then simply denote $h^{\beta, \omega}$ by $h^{\beta}$.

We now wish to prove that $h^{\beta}=\beta \star \partial_{x} H$ in the distribution sense.

Let $\alpha(x) \in \mathcal{D}(\mathbb{R})$ be such that $\alpha \equiv 1$ on $\left[-R_{1}, R_{1}\right]$. Let $\eta(x) \in \mathcal{D}(\mathbb{R})$; we have

$$
\begin{aligned}
\left\langle\left(\beta \star \partial_{x} H_{\epsilon}\right)_{x}, \eta(x)\right\rangle & =\left\langle\beta_{y} \otimes\left(\partial_{x} H_{\epsilon}\right)_{x}, \eta(y+x)\right\rangle \\
& =\left\langle\beta_{y} \otimes\left(\partial_{x} H_{\epsilon}\right)_{x},(\alpha(x) \eta(y+x))\right\rangle \\
& =\int_{y \in \mathbb{R}} \beta(y) \int_{\mathbb{R}} \alpha(x) \eta(y+x) \partial_{x} H_{\epsilon} d x d y \\
& =-\int_{y \in K_{\beta}} \beta(y)\left\langle H_{\epsilon}, \partial_{x}(\alpha(x) \eta(y+x))\right\rangle d y .
\end{aligned}
$$

The notation $\left\langle T_{x}, \eta(y+x)\right\rangle$ above simply refers to the fact that the distribution $T$ is applied to the function $\theta(x)=\eta(y+x)$. It is not to be confused with the partial derivative with respect to $x$, which we denote throughout this paper by $\partial_{x} T$.

Thanks to the uniform boundedness of $G_{\epsilon}$ in $L^{1}\left(\Omega_{g}\right)$ (see the beginning of Step 4.1) and the properties of the extension operator $P$, the expression

$$
\left\langle H_{\epsilon}, \partial_{x}(\alpha(x) \eta(y+x))\right\rangle=\int H_{\epsilon} \partial_{x}(\alpha(x) \eta(y+x)) d x
$$


is uniformly (in $\epsilon$ ) bounded. On the other hand Theorem 4.5 shows that

$$
\begin{aligned}
\int_{\Omega_{g}} G_{\epsilon} \partial_{x}(\alpha(x) \eta(y+x)) d x & =\int_{\Omega_{g} \times V \times(0, T)} g_{\epsilon} a(v) \varphi(v) \phi(t) \partial_{x}(\alpha(x) \eta(y+x)) d v d t d x \\
\rightarrow \epsilon \rightarrow 0 & \int_{\Omega_{g} \times V \times(0, T)} g a(v) \varphi(v) \phi(t) \partial_{x}(\alpha(x) \eta(y+x)) d v d t d x \\
& =\int_{\Omega_{g}} G \partial_{x}(\alpha(x) \eta(y+x)) d x .
\end{aligned}
$$

Hence using the explicit definition of the extension operator $P$, we have

$$
\left\langle H_{\epsilon}, \partial_{x}(\alpha(x) \eta(y+x))\right\rangle=\int_{\mathbb{R}} H_{\epsilon} \partial_{x}(\alpha(x) \eta(y+x)) d x
$$

converges as $\epsilon \rightarrow 0$ to

$$
\int_{\mathbb{R}} H \partial_{x}(\alpha(x) \eta(y+x)) d x=\left\langle H, \partial_{x}(\alpha(x) \eta(y+x))\right\rangle .
$$

Therefore, since $\beta \in L^{1}(\mathbb{R})$ with compact support, we can use the dominated convergence theorem to conclude that the right-hand side in (88) converges to

$$
\begin{aligned}
-\int_{y \in K_{\beta}} \beta(y)\left\langle H, \partial_{x}(\alpha(x) \eta(y+x))\right\rangle d y & =\int_{y \in \mathbb{R}} \beta(y)\left\langle\partial_{x} H,(\alpha(x) \eta(y+x))\right\rangle d y \\
& =\left\langle\left(\beta \star \partial_{x} H\right)_{x}, \eta(x)\right\rangle .
\end{aligned}
$$

On the other hand, using the distributional definition of the convolution for $\beta \star \partial_{x} H_{\epsilon}$ and the fact that $\beta \star \partial_{x} H_{\epsilon}$ converges to $h^{\beta}$ in $L^{1}(\mathbb{R})$, together with (89), we obtain

$$
\left\langle h^{\beta}, \eta\right\rangle=\left\langle\beta \star \partial_{x} H, \eta\right\rangle .
$$

This shows that the convolution (of two distributions) $\beta \star \partial_{x} H$ is a function in $L^{1}(\mathbb{R})$ equal to $h^{\beta}$. Hence for any $\beta \in L^{1}(\mathbb{R})$ with compact support we have proved that $\beta \star \partial_{x} H_{\epsilon}$ converges in $L^{1}(\mathbb{R})$ to $h^{\beta}=\beta \star \partial_{x} H$.

4.4. We prove that the distribution $\partial_{x} H$ is actually a measure.

Step 4.3 above implies in particular that for any $\beta \in L^{1}(\mathbb{R})$ with compact support, $\beta \star \partial_{x} H$ is a function in $L^{1}(\mathbb{R})$. Hence $\beta \star \partial_{x} H$ defines a measure on $\mathbb{R}$.

We shall prove that the distribution $\partial_{x} H$ is actually a measure. This results from the fact that if $T$ is a distribution such that $\forall \beta \in L^{1}(\mathbb{R})$ with compact support, $T \star \beta$ is a measure, then $T$ is itself a measure [17].

4.5. We prove that

$$
\begin{gathered}
\operatorname{limit}_{\epsilon \rightarrow 0} \int_{\Omega_{g}} \tilde{\psi}(y)\left(\partial_{x} G_{\epsilon}(y)-\partial_{x} G(y)\right) d y=0 \\
\forall \tilde{\psi} \text { continuous bounded function on } \Omega_{g} .
\end{gathered}
$$

In the previous steps 4.3 and 4.4 , we proved that $h^{\beta}=\beta \star \partial_{x} H$ in the classical sense and that $\partial_{x} H$ is a measure. Denote $\mu$ as the measure $\mu=\partial_{x} H$. Then we know from the definition of the convolution of a function and a measure that the function $\beta \star \mu(x)=\beta \star \partial_{x} H$ is given by

$$
\beta \star \mu(x)=\int_{\mathbb{R}} \beta(x-y) d \mu(y)=\int_{\mathbb{R}} \beta(x-y) \partial_{x} H(y) d y .
$$


(Recall that $\beta$ is of compact support.) In the previous Steps 4.3 and 4.4, we proved that for any $\beta \in L^{1}(\mathbb{R})$ with compact support, we have $\beta \star \partial_{x} H_{\epsilon} \rightarrow_{\epsilon \rightarrow 0} h^{\beta}$ in $L^{1}(\mathbb{R})$. In other words, we have

$$
\begin{aligned}
& \operatorname{limit}_{\epsilon \rightarrow 0} \int_{\mathbb{R}}\left|\beta \star \partial_{x} H_{\epsilon}-h^{\beta}\right| d x \\
= & \operatorname{limit}_{\epsilon \rightarrow 0} \int_{\mathbb{R}}\left|\beta \star \partial_{x} H_{\epsilon}-\beta \star \partial_{x} H\right| d x \\
= & \operatorname{limit}_{\epsilon \rightarrow 0} \int_{\mathbb{R}}\left|\int_{\mathbb{R}} \beta(x-y) \partial_{x} H_{\epsilon}(y) d y-\int_{\mathbb{R}} \beta(x-y) \partial_{x} H(y) d y\right| d x \\
= & 0 .
\end{aligned}
$$

Then

(93) $\operatorname{limit}_{\epsilon \rightarrow 0}\left(\int_{\mathbb{R}} \beta(x-y) \partial_{x} H_{\epsilon}(y) d y-\int_{\mathbb{R}} \beta(x-y) \partial_{x} H(y) d y\right)=0$, for a.e. $x \in \mathbb{R}$.

Let $x=\gamma$ be a point at which such limit exists and is 0 . Let $\tilde{\psi}(y)$ be a continuous bounded function on $\bar{\Omega}_{g}=[l, 1]$. Let $\beta(z)=\tilde{\psi}(\gamma-z)$ for $(\gamma-z) \in[l, 1]$ and $\beta(z)=0$ otherwise. Then $\beta \in L^{1}(\mathbb{R})$ and has a compact support. Therefore, plugging this $\beta$ in (93), we obtain

$$
\operatorname{limit}_{\epsilon \rightarrow 0} \int_{\Omega_{g}} \tilde{\psi}(y)\left(\partial_{x} H_{\epsilon}(y)-\partial_{x} H(y)\right) d y=0 .
$$

Now the definition of the extension operator [3] yields $\partial_{x} H_{\epsilon}(y)=\partial_{x} G_{\epsilon}(y)$ and $\partial_{x} H(y)=\partial_{x} G(y)$ on $\Omega_{g}$. Thus, we obtain (91).

4.6. Now combining Step 4.5, Theorem 4.5(iii), and (85)-(86), we conclude that

$$
\operatorname{limit}_{\epsilon \rightarrow 0} \int_{\Omega_{g} \times V \times(0, T)} \tilde{\psi}(y) \partial_{v} \varphi(v) \phi(t) d m_{\epsilon}=\int_{\Omega_{g} \times V \times(0, T)} \tilde{\psi}(y) \partial_{v} \varphi(v) \phi(t) d m
$$

(95) $\forall \tilde{\psi}$ continuous bounded function on $\Omega_{g}$.

In particular, (95) is valid for any function $\tilde{\psi} \in C_{0}^{1}\left(\bar{\Omega}_{g}\right)$. Using this, we conclude that the expression in (83) converges to the expression in (79) for any $\varphi(v) \in C_{0}^{1}(V)$ with $\varphi \equiv 0$ on $V \backslash V^{-}$and any $\psi \in C_{0}^{1}\left(\bar{\Omega}_{g} \times(0, T)\right)$ of the form $\psi(x, t)=\tilde{\psi}(x) \phi(t)$. By the remark at the beginning of Step 4, this convergence takes place for all functions $\varphi(v) \in C_{0}^{1}(V)$ with $\varphi \equiv 0$ on $V \backslash V^{-}$and all functions $\psi(x, t) \in C_{0}^{1}\left(\bar{\Omega}_{g} \times(0, T)\right)$. This concludes the proof of our claim.

Step 5. Here we conclude the proof.

Using the above convergence in (84) and (80), we obtain

$$
\begin{aligned}
& \operatorname{limit}_{\epsilon \rightarrow 0} \int_{\Gamma_{i}^{-} \times(0, T)} a(v) \cdot n \varphi \psi g_{\epsilon} \\
& =\operatorname{limit}_{\epsilon \rightarrow 0} \int_{\Gamma_{i} \times V^{-\times}(0, T)} a(v) \cdot n \varphi \psi g_{\epsilon}
\end{aligned}
$$

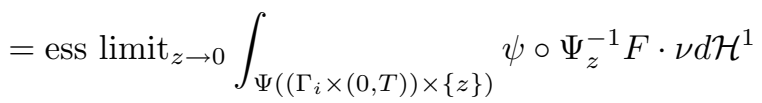

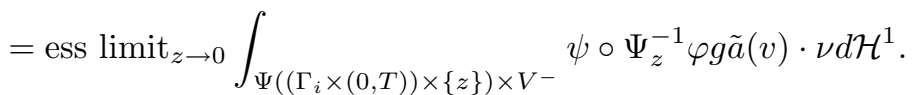


Since $\varphi \in C_{0}^{1}(V)$ and $\psi \in C_{0}^{1}\left(\bar{\Omega}_{g} \times(0, T)\right)$ are arbitrary functions with $\varphi \equiv 0$ on $V \backslash V^{-}$and $\psi \equiv 0$ on $\partial\left(\Omega_{g} \times(0, T)\right) \backslash\left(\Gamma_{i} \times(0, T)\right)$, (96) and (82) show that $\tilde{g}$ is the weak trace of $g$ on $\Gamma_{i}^{-} \times(0, T)=\Gamma_{i} \times V^{-} \times(0, T)$. Moreover, this weak trace satisfies (81), which therefore yields the theorem.

\section{Analysis of the Coupled Kinetic/COnSERVATion laWs System}

In this final section, we state a result about the existence of an entropic solution to the coupled kinetic/conservation laws system (8)-(13) in the sense of Definition 3.1 .

Theorem 5.1. Assume that

$$
\begin{aligned}
& \left\|f_{\epsilon 0}\right\|_{L^{\infty}\left(\Gamma_{1}^{-} \times[0, T]\right)}<C_{1},\left\|g_{\epsilon}^{0}\right\|_{L^{\infty}\left(\Omega_{g} \times V\right)}<C_{2}, \quad\left\|f_{\epsilon}^{0}\right\|_{L^{\infty}\left(\Omega_{l} \times V\right)}<C_{3}, \\
& \left\|g_{\epsilon 1}\right\|_{L^{\infty}\left(\Gamma_{2}^{-} \times[0, T]\right)}<C_{4},\left\|f_{\epsilon}^{0}\right\|_{L^{1}\left(\Omega_{l} \times V\right)}<C_{5},\left\|g_{\epsilon}^{0}\right\|_{L^{1}\left(\Omega_{g} \times V\right)}<C_{6}, \\
& \left\|a(v) \cdot n_{l} f_{\epsilon 0}\right\|_{L^{1}\left(\Gamma_{1}^{-} \times(0, T)\right)}<C_{7}, \quad\left\|a(v) \cdot n_{g} g_{\epsilon 1}\right\|_{L^{1}\left(\Gamma_{2}^{-} \times(0, T)\right)}<C_{8}
\end{aligned}
$$

with $C_{i}, i=1, \cdots, 8$, positive constants independent of $\epsilon$.

Assume also that the initial and boundary data $f_{\epsilon}^{0}, f_{\epsilon 0}, g_{\epsilon}^{0}$, and $g_{\epsilon 1}$ are compactly supported in $v \in V$ with supports included in a fixed compact set independent of $\epsilon$. Finally assume that (48) holds and that as $\epsilon \rightarrow 0$,

$$
\begin{aligned}
& \left\|g_{\epsilon}^{0}(\cdot, \cdot)-\chi_{w^{0}(\cdot)}(\cdot)\right\|_{L_{l o c}^{1}\left(\Omega_{g} \times L^{1}(V)\right)} \rightarrow 0, \\
& f_{\epsilon}^{0} \rightarrow f^{0} \text { strongly in } L^{1}\left(\Omega_{l} \times V\right), \\
& a(v) \cdot n f_{\epsilon 0} \rightarrow a(v) \cdot n f_{0} \text { weakly in } L^{1}\left(\Gamma_{1}^{-} \times(0, T)\right), \\
& a(v) \cdot n g_{\epsilon 1} \rightarrow a(v) \cdot n g_{1}=a(v) \cdot n \chi_{w_{1}} \text { strongly in } L^{1}\left(\Gamma_{2}^{-} \times(0, T)\right) .
\end{aligned}
$$

Then there exists an entropic solution to the system (8)-(13) in the sense of Definition 3.1 .

Remark 5.1. Following Remark 4.3, Theorem 4.8 is valid also when the initial and boundary data are no longer compactly supported in $v$. Therefore by the use of Theorem 3.1 on the equivalence between the kinetic and the entropic formulations and Theorem 4.8, Theorem 5.1 is also valid when the boundary and initial data are not necessarily compactly supported in $v$.

Proof of Theorem 5.1. This theorem is an immediate consequence of Theorem 4.8 and the kinetic formulation of the coupled system (8)-(13) given in Theorem 3.1

\section{REFERENCES}

1. Bardos C., Golse F., Perthame B., and Sentis R., The nonaccretive radiative transfer equations: existence of solutions and Rosseland approximations, J. Funct. Anal. 77, 434-460 (1988). MF0933978 (89f:35174)

2. Bardos C., Leroux A.-Y., and Nedelec J.-C., First order quasilinear equations with boundary conditions, Comm. PDE 4 (9), 1017-1034 (1979). MR.0542510(81b:35052)

3. Brezis, H., Analyse functionnelle Théorie et applications, second edition, Collection Mathématiques appliquées pour la maîtrise sous la direction de P. G. Ciarlet et J. L. Lions, Masson, Paris, 1987. MR0697382 (85a:46001)

4. Chen G.-Q. and Frid H., On the theory of divergence-measure fields and its applications, Boletin da Sociedade Brasileira de Matematica, to appear.

5. Chen G.-Q. and Perthame B., Well-posedness for anisotropic degenerate parabolic-hyperbolic equations. Annales de l'institut Henri Poincaré: Analyse Nonlinéaire, 2002, to appear. 
6. Dautray, R and Lions, J.-L., Analyse mathématique et calcul numérique pour les sciences et les techniques, Volume 9, Evolution: numérique, transport. Masson, Paris, 1988. MR 1016606 (90m:00006c)

7. Diperna, R. J., Lions, P.-L. and Meyer, Y., $L^{p}$ regularity of velocity averages, Ann. Inst. Henri Poincaré, 8, Nos 3-4, pp. 271-287, 1991. MR1127927 (92g:35036)

8. Evans, L. C. and Gariepy, R. F., Measure Theory and Fine Properties of Functions, Studies in advanced mathematics, CRC Press, 1992. MR1158660 (93f:28001)

9. Golse F., Lions P.-L., Perthame B., and Sentis R., Regularity of the moments of the solution of a transport equation, J. Funct. Anal. 16 (1988). MR0923047|(89a:35179)

10. Krushkov S. N., First order quasilinear equations with several independent variables, Math. Sbornik. 81 (123), 228-255; Math USSR Sbornik 10, 217-243 (1970). MR0267257 (42:2159)

11. Lions, P.-L., Perthame B. and Souganidis P.E., Existence of entropy solutions for the hyperbolic systems of isentropic gas dynamics in Eulerian and Lagrangian coordinates, C.P.A.M. Vol. 49 (1996) 599-638. MR1383202 (97e:35107)

12. Lions, P.-L., Perthame B. and Tadmor E., A kinetic formulation of multidimensional scalar conservation laws and related equations, J. AMS, 7, No. 1, 169-190, 1994 . MR1201239 (94d:35100)

13. Lions, P.-L., Perthame B. and Tadmor E., Kinetic formulation of the isentropic gas dynamics and $p$-systems, Comm. Math. Phys. 163 (1994) 415-431. MR1284790 (95c:76093)

14. Perthame B., Uniqueness and error estimates in first order quasilinear conservation laws via the kinetic entropy defect measure, J. Math. Pures Appl., 77, 1998, pp. 1055-1064. MR1661021 (2000e:35141)

15. Perthame B., Kinetic formulations of conservation laws, Oxford Lecture Series in Mathematics and its Applications, vol. 21, Oxford University Press, Oxford, 2002. MR2064166

16. Perthame B. and Tadmor E., A kinetic equation with kinetic entropy functions for scalar conservation laws, Comm. Math. Phys. 136, 501-517 (1991). MR.1099693 (92d:82095)

17. Schwartz L., Théorie des distributions, Hermann, Paris, 1978. MR.0209834 (35:730)

18. Tartar L., Une nouvelle méthode de résolution d'équations aux dérivées partielles nonlinéaires, Lecture Notes in Math., vol. 665, Springer, Berlin, 1977, pp. 228-241. MF0519433 (80b:35007)

19. Tartar L., Compensated compactness and applications to partial differential equations. In: Research Notes in Mathematics, vol. 39, Nonlinear Analysis and Mechanics, Heriot-Watt Sympos. vol. 4, Knopps, R. J. (ed.) pp. 136-211. Boston, London: Pitman Press, 1975. MR0584398 (81m:35014)

20. Tidriri M., Asymptotic analysis of a coupled system of kinetic equations. C. R. Acad. Sci. Paris, t. 328, Série I Math., 637-642, 1999. MR:1680029|(99m:76099)

21. Tidriri M. D., Numerical analysis of coupling for a kinetic equation. Mathematical Modelling and Numerical Analysis M2AN, Vol. 33, No 6, 1999, p. 1121-1134. MR1736892 (2000j:76128)

22. Tidriri M. D., A novel class of multiscale models in mathematical physics. J. Nonlinear Analysis, Vol. 47 (2001) 4995-5008. MR1975891 (2004d:35039)

23. Tidriri M. D., Rigorous derivation and analysis of coupling of kinetic equations and their hydrodynamics limits for a simplified Boltzmann model. J. Stat. Phys. Vol. 104. Nos. 1/2 (2001), pp. 257-292. MR1851389 (2002h:82075)

24. Tidriri M. D., New models for the solution of intermediate regimes in transport theory and radiative transfer: existence theory, positivity, asymptotic analysis, and approximations. J. Stat. Phys., Vol. 104. Nos. 1/2 (2001), pp. 293-328. MR1851390(2002j:82104)

25. Tidriri M. D., Analysis of a coupled system of kinetic equations and their hydrodynamic (conservation laws) limit: rigorous derivation and existence theory via BV theory. Accepted.

26. Tidriri M., Hydrodynamic limit of a B.G.K. like model on domains with boundaries and analysis of kinetic boundary conditions for scalar multidimensional conservation laws. J. Stat. Phys., Vol. 115. Nos. 5/6 (2004), pp. 1715-1754. MR2066297

27. Tidriri M., Hydrodynamic limit of a B.G.K. kinetic model and kinetic formulation of conservation laws on domains with boundaries. To appear in Archive for Rational Mechanics and Analysis.

Department of Mathematics, Iowa State University, Ames, Iowa 50011-2064

E-mail address: tidriri@iastate.edu 